\title{
Laboreal
}

Volume $16 \mathrm{~N}^{\circ} 2$ | 2020

Programa de Pesquisa do Curso da Ação

\section{Simulação multiagente da atividade humana: uma concretização em ergonomia do programa de pesquisa tecnológica "curso de ação"}

Simulación multiagente de la actividad humana: una concreción en ergonomía del programa de investigación tecnológico "curso de acción"

Simulation multi-agent de l'activité humaine : une concrétisation en ergonomie du programme de recherche technologique "cours d'action"

Multi-agent simulation of human activity: a concretization in ergonomics of the "course of action" technological research program

\section{Yvon Haradji}

\section{(2) OpenEdition}

\section{Journals}

Edición electrónica

URL: http://journals.openedition.org/laboreal/16587

DOI: $10.4000 /$ laboreal. 16587

ISSN: 1646-5237

Editor

Universidade do Porto

Referencia electrónica

Yvon Haradji, « Simulação multiagente da atividade humana: uma concretização em ergonomia do programa de pesquisa tecnológica "curso de ação" », Laboreal [En línea], Volume 16 N² | 2020,

Publicado el 01 diciembre 2020, consultado el 13 diciembre 2020. URL : http://

journals.openedition.org/laboreal/16587 ; DOI : https://doi.org/10.4000/laboreal.16587

Este documento fue generado automáticamente el 13 diciembre 2020.

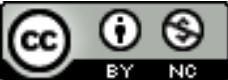

Laboreal está licenciado com uma Licença Creative Commons - Atribuição-NãoComercial 4.0 Internacional. 


\section{Simulação multiagente da atividade humana: uma concretização em ergonomia do programa de pesquisa tecnológica "curso de ação"}

Simulación multiagente de la actividad humana: una concreción en ergonomía del programa de investigación tecnológico "curso de acción"

Simulation multi-agent de l'activité humaine : une concrétisation en ergonomie du programme de recherche technologique " cours d'action " Multi-agent simulation of human activity: a concretization in ergonomics of the "course of action" technological research program

\section{Yvon Haradji}

\section{NOTA DEL EDITOR}

Manuscrito recibido en: 02/03/2020

Aceptado tras peritaje en: 26/10/2020

Traducción : Susana Martha Gurovich [susanamarthag@gmail.com]

Estos trabajos no hubieran sido posibles de no haber tenido innumerables contribuciones de investigación y de ingeniería. En primer lugar, desde luego, diferentes responsables de la R\&D (I\&D- Investigación \& Desarrollo) de EDF (Électricité de France) hicieron posible estos trabajos que no siempre estaban en el espíritu de la época. Desde el punto de vista técnico, la idea de una investigación multiagente fue elaborada con Alexis Drogoul; y siguió principalmente con Nicolas Sabouret, François Sempé, Quentin Raynaud, Thomas Huraux et Jérémy Albouys-Perrois. Christian Inard et Benoit Charrier realizan en la actualidad la necesaria articulación con una problemática del sistema térmico de la construcción. Por el lado de la ergonomía, la idea maduró en el seno de la red ACT'ING pero se concretó con Mariane Galbat, Julien Guibourdenche y 
Germain Poizat. Elise Prieur en diseño y Bruno Delenne para las curvas de carga fueron indispensables cuando hubo que validar nuestros trabajos. Finalmente, Mathieu Schumann ha sido un actor comprometido con los sistemas térmicos de la construcción, determinante en momentos difíciles; en la actualidad, es quien lleva adelante el proyecto.

\section{Introducción}

1 Este documento se basa en los trabajos de investigación de ayer y de hoy sobre la simulación de la actividad humana y los consumos en el hábitat (SMACH [1]). SMACH se transformó en un servicio destinado a los expertos en energía (técnicos termoeléctricos, expertos en ofertas arancelarias, en curvas de consumo, en vehículos eléctricos, etc.) con el deseo de anticipar una situación futura: que los datos realistas producidos por SMACH los ayuden a calcular los efectos de sus innovaciones sobre el consumo de energía. SMACH es también un conjunto de acciones de investigación y de ingeniería que apuntan a hacer que la simulación evolucione al ritmo de las transformaciones principales y actuales del mundo de la energía (el autoconsumo colectivo, el vehículo eléctrico, etc.).

2 La plataforma SMACH es el resultado de una construcción progresiva, a lo largo de muchos años (desde 2007 hasta 2019). Su originalidad, desde el comienzo, radica en un principio de diseño muy estructurante: la primacía acordada a la actividad humana. Consideramos que el consumo de energía se inscribe en un universo técnico (la construcción, los equipamientos), pero es, en primer lugar, el resultado de la actividad humana en el hábitat. Es la dinámica de la vida cotidiana de las personas en su hogar la que estructura la dinámica del ecosistema simulado. Nunca hubiéramos considerado esta orientación primera si no hubiésemos conocido las posibilidades conceptuales y técnicas de los sistemas multiagentes (SMA). Esta corriente de la Inteligencia Artificial se apoya en algunos principios simples: un enfoque distribuido, la autonomía de los agentes, un lugar fundamental asignado a la interacción y un diseño de la urgencia como manifestación de la organización colectiva de los agentes (Ferber, 1995). Los SMA ya fueron implementados en ergonomía para concebir sistemas cooperativos complejos (Dugdale, Pavard, \& Soubie, 2000) y permitieron orientar las decisiones relativas a los colectivos profesionales del SAMU (Service d'aide médicale urgente - Servicio de Ayuda Médical Urgente), del control aéreo (Pavard, 2002). Era pues casi natural recurrir a esos sistemas para simular la vida cotidiana en este hábitat para problemáticas de eficiencia energética en el hábitat.

En este artículo, nos planteamos reconstruir la historia de esta investigación en nuestro contexto industrial. Nuestro objetivo es proponer una mirada reflexiva sobre nuestra práctica de investigación con el fin de caracterizar lo que puede ser, en ergonomía, un programa de investigación tecnológico "curso de acción" (Durand, 2008; Theureau, 2006, 2009b). En tal sentido, presentamos un análisis longitudinal de dicha investigación con la ambición principal de mostrar cómo la relación orgánica con la actividad humana estructura un programa de investigación tecnológico. Asimismo, describiremos la dinámica interna de la investigación, los motores de su crecimiento, la calidad reconocida y que culmina con la creación de un servicio para los expertos en energía. Para hacerlo, nos apoyaremos en la descripción de las tres etapas de la investigación SMACH, en esos momentos particulares de incertidumbre y de investigación que dieron lugar a esta innovación. Este artículo puede leerse como un 
alegato en favor de un fortalecimiento de la relación orgánica entre diseño y análisis de la actividad en ergonomía, pero también como una base para generalizar y precisar los criterios efectivos de validación de un programa de investigación tecnológico.

\section{Un programa de investigación tecnológico sobre la simulación de la actividad humana}

\subsection{La simulación SMACH para anticipar los effectos de la actividad humana sobre los consumos de energía en el hábitat}

4 La simulación es una herramienta metodológica conocida y reconocida en ergonomía. Su función es muy a menudo la de anticipar una situación futura. Se simula el entorno de trabajo (espacio de trabajo transformado, maqueta arquitectónica o interactiva, simulador en escala 1:1, etc.) y en ese entorno se pone en escena la actividad de los operadores (Béguin \& Weill-Fassina, 1997; Van Belleghem, 2018). Estos autores utilizan principalmente la simulación para implicar a operadores en el diseño de su situación de trabajo futura según los principios del diseño participativo. Nuestro objetivo con la simulación es simular situaciones en las cuales la actividad humana tiene un impacto sobre un sistema técnico-organizacional-cultural futuro que habrá que concebir (Kashif, Ploix, Dugdale, \& Le, 2013). La simulación es aquí un medio para responder al tan conocido problema de la paradoja de la ergonomía de diseño (Pinsky \& Theureau, 1984): decir algo realmente fundado acerca de una situación de trabajo es posible cuando ésta ha sido diseñada completamente, pero entonces ya es demasiado tarde para intervenir en el diseño. La simulación favorece la anticipación, puesto que funciona como un "laboratorio virtual": "abre el espacio de diseño" y nutre la interacción (debate y negociación) entre los actores involucrados en el diseño (Barcellini, Van Belleghem, \& Daniellou, 2013).

5 La simulación SMACH se inscribe en esta perspectiva de anticipación de una situación futura: es una herramienta de ayuda para que los diseñadores puedan responder a problemáticas de eficiencia energética con decisión e imaginación (Guibourdenche, Salembier, Poizat, Haradji, \& Galbat, 2007), en situaciones que no existen aún (autoconsumo colectivo, vehículo eléctrico...). Se pueden así representar hipótesis de transformación de la actividad humana para anticipar el impacto de las innovaciones tecnológicas sobre el consumo de energía. Es una plataforma utilizable para estudios que abarcan desde un hogar hasta una población. La simulación pone en escena a agentes informáticos que organizan su vida cotidiana individual y colectiva en un hábitat digital. Generan consumos de electricidad debido a sus actividades cotidianas con aparatos eléctricos, a su percepción del confort y a una preocupación más o menos acentuada por el rendimiento energético. El funcionamiento general de SMACH es el siguiente:

- Los datos de base de SMACH corresponden a una descripción estática de un hogar e incluyen la cantidad de personas en el hogar, la edad, el sexo, las acciones posibles, las preferencias en cuanto a confort, los equipamientos, el tipo de hábitat. Esos datos surgen de encuestas, principalmente del INSEE (Institut National de la Statistique et des Études ÉconomiquesInstituto nacional de Estadística y Estudios Económicos).

- El motor de actividad humana corresponde al motor multiagente que, al inicio de la simulación, hará que interactúen todos los elementos de descripción del hogar que se está 
simulando. En cada espacio de tiempo (equivalente a un minuto de simulación), los agentes pueden realizar acciones individuales, interactuar con los otros agentes de la situación (los miembros del hogar) o con el entorno (el hábitat, la calefacción, etc.).

De esta manera, distinguimos en la simulación (figura 1) lo que pertenece al orden del encuadre estático de la simulación (los datos de base del hogar) y lo que pertenece al orden de la dinámica de los agentes (el motor de actividad humana).

Figura 1: Lógica de funcionamento de SMACH

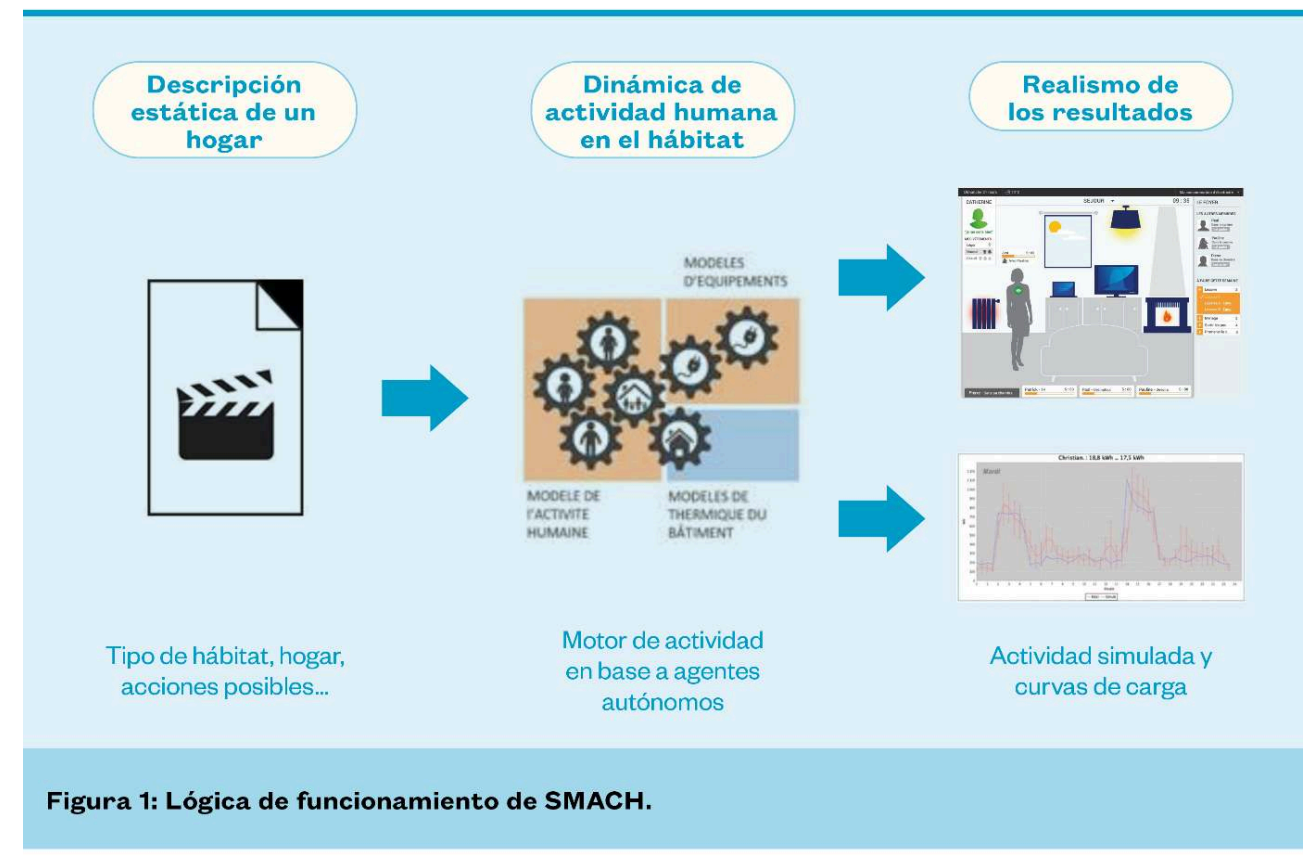

Figura 1: Lógica de funcionamento de SMACH

7 En la plataforma SMACH se asocian diferentes IHM [2²] a fin de poner la simulación a disposición de usuarios con perfiles y objetivos de uso muy variados (expertos en energía, habitantes, visitantes en los showroom).

\subsection{El programa de investigación tecnológico del curso de acción}

Leonardo Pinsky proponía, en su presentación en el XXVI Congreso de la Sociedad de Ergonomía de Lengua Francesa (Pinsky, 1990a), desarrollar una investigación tecnológica, una teoría de la práctica. En dicha intervención, Pinsky precisa, en primer lugar, que una investigación tecnológica no es reductible a una ciencia, a una disciplina, pues, citando a Koyré (Koyré, 1971), señala que el pensamiento práctico es esencialmente diferente del pensamiento teórico, científico; y que se distingue de éste por la autonomía que se manifiesta en la inventiva y la creatividad de las que da muestras. Menos aún puede reducirse a una ciencia si se tiene en cuenta que para su diseño es fundamentalmente el resultado de una interdisciplina. Pinsky se sitúa en la prolongación del diseño centrado en el usuario (Norman \& Draper, 1986; Pinsky, 1990b) y considera que la acción de los ergónomos en el diseño pasa por la realización de un modelo práctico, es decir, un modelo cuyo objetivo es la acción de transformación en base al diagnóstico/pronóstico que el ergónomo hace de la situación. Asimismo señala que el diseño de una situación nueva necesita criterios para orientarla. Para él, uno de 
esos criterios es la carga de trabajo, que permite orientar la investigación tecnológica en el sentido de mejorar las condiciones de realización de la actividad. El autor pone mucho cuidado en señalar que la carga de trabajo no es para él una noción científica sino una noción tecnológica útil: "Así como la "carga de trabajo" como noción científica, cuantificable, o hasta solo formalizable es una aberración que la ergonomía descartó con razón, así también la "carga de trabajo" como noción tecnológica, informada a medida que progresaba la investigación científica, es según nuestro entender, para retener e incluso, para desarrollar" (Pinsky, 1990a, p8, traducción libre). Este texto y el de 1987 (Pinsky \& Theureau, 1987) son, como se dijo en la introducción de este artículo, textos fundacionales, que sientan las bases de lo que entendemos por investigación tecnológica: una investigación sobre la práctica de diseño, interdisciplinaria, basada en el conocimiento de la actividad humana (y especialmente del curso de acción) y que integra, muy desde el comienzo del diseño, el diagnóstico/ pronóstico [ $\left.{ }^{3}\right]$ del ergónomo, a través de la modelización, para contribuir directamente al diseño de las situaciones futuras.

El programa del curso de acción distinguió y articuló muy tempranamente dos programas de investigación: "el programa de investigación empírica del curso de acción" que se ocupa del conocimiento científico de la actividad y "el programa de investigación tecnológico del curso de acción" relativo al conocimiento producido a partir de la práctica de diseño. Tomando como referencia a los autores (Durand, 2008; Pinsky \& Theureau, 1987; Theureau, 2006, 2019), definimos a continuación un programa de investigación tecnológico (PIT) del curso de acción:

- Un PIT, como toda investigación tecnológica, no es la aplicación de una ciencia (una ciencia aplicada), sino que resulta de una influencia recíproca entre conocimientos teóricos y empíricos sobre la actividad humana y conocimientos teóricos y técnicos vinculados al diseño de las situaciones. El PIT es entonces el resultado de una relación orgánica con uno o varios programas de investigación empíricos sean éstos o no del curso de acción.

- Inicialmente, el objeto de diseño de un PIT estaba enfocado en el tema de la ayuda a la actividad humana. El diseño no apunta a artefactos que se substituyan al operador; su objetivo es más bien concebir una ayuda para el usuario/operador en la interacción, en sus decisiones de acción, en la evaluación de sus acciones (Jeffroy \& Lambert, 1992; Pinsky \& Theureau, 1987). El usuario/operador actúa y transforma la situación a partir de su punto de vista, de su flujo de interacciones asimétricas; el diseño en términos de ayuda consiste en concebir un entorno (técnico, organizacional...) que favorecerá su interacción asimétrica en la situación.

- El objeto de diseño se fue ampliando progresivamente. Del diseño de un sistema de ayuda pasó a ser el diseño de una situación apropiable (Poizat, Durand, \& Theureau, 2016; Theureau, 2009b). Diseñar es anticipar una situación futura considerando todos los efectos que ésta puede llegar a producir sobre el operador, es construir un entorno (técnico, cultural, organizacional...) que se inscriba en el proceso continuo de transformación de la actividad. Theureau (Theureau, 2011) señala que el diseño de la apropiabilidad debe referirse a la apropiación de la situación (el acoplamiento del actor en actividad con el entorno y los otros actores), la apropiación en el cuerpo (ser uno con...) y la apropiación cultural (relativa al sistema de valores del usuario/operador).

- Un PIT puede ser evaluado en virtud de dos criterios. El primero es el de su poder heurístico, es decir su capacidad para responder a necesidades, para poner en evidencia nuevas necesidades, para resistir a la objeción de los actores del diseño, para plantear nuevos interrogantes de diseño. El segundo criterio es el de su capacidad de crecimiento, es decir, su 
capacidad de ampliar su campo inicial, su apertura/desarrollo a otros campos, su incidencia en términos de nuevos interrogantes formulados a los programas de investigación empírica que tienen una relación orgánica con el PIT.

- Finalmente, escogemos también de esos autores el criterio primordial de la eficacia técnicoorganizacional cultural que ofrece criterios e índices de calidad tecnológica (como por ejemplo, la utilidad, la utilizabilidad, la carga de trabajo...) y una instancia de test/validación de esa eficacia.

10 En este documento, nos apoyaremos en la noción de programa de investigación tecnológico (PIT) para explicar el proceso que implementamos para concebir la simulación SMACH. Esta experiencia de diseño nos servirá como base para explicar qué es un programa de investigación tecnológico. Procuraremos mostrar cómo construimos el PIT SMACH en una relación orgánica con la actividad humana y con qué criterio evaluamos su calidad tecnológica, su poder heurístico y su capacidad de crecimiento. Basándonos en la experiencia SMACH, no se tratará de abordar la cuestión del PIT en sus aspectos generales (PIT genérico del curso de acción), sino más bien de reflexionar a partir de un PIT específico al que llamamos "simular la actividad humana in silico".

\subsection{El programa de investigación tecnológico SMACH en varias etapas}

11 La investigación SMACH necesitó innovar desde un punto de vista conceptual (las relaciones entre modelos de actividad y multiagentes), metodológico (la validación cualitativa y cuantitativa) y técnico (el desarrollo de la plataforma multiagente). Se trata más precisamente de un programa de investigación tecnológico [ $\left.{ }^{4}\right]$ (PIT) específico para la simulación de la actividad humana in silico. Fue elaborado progresvamente tomando como base una relación estructural entre una investigación empírica sobre el conocimiento de la actividad humana en la vida cotidiana en el hábitat y el diseño informática de una simulación de la actividad humana y de sus consumos en el hábitat. Respecto a la simulación SMACH, desarrollamos esta lógica general de diseño para las dos partes constitutivas de la simulación: la IHM y el motor de simulación (ver figura 2). 
Figura 2: Los dos ejes del diseño centrada en la actividad em SMACH

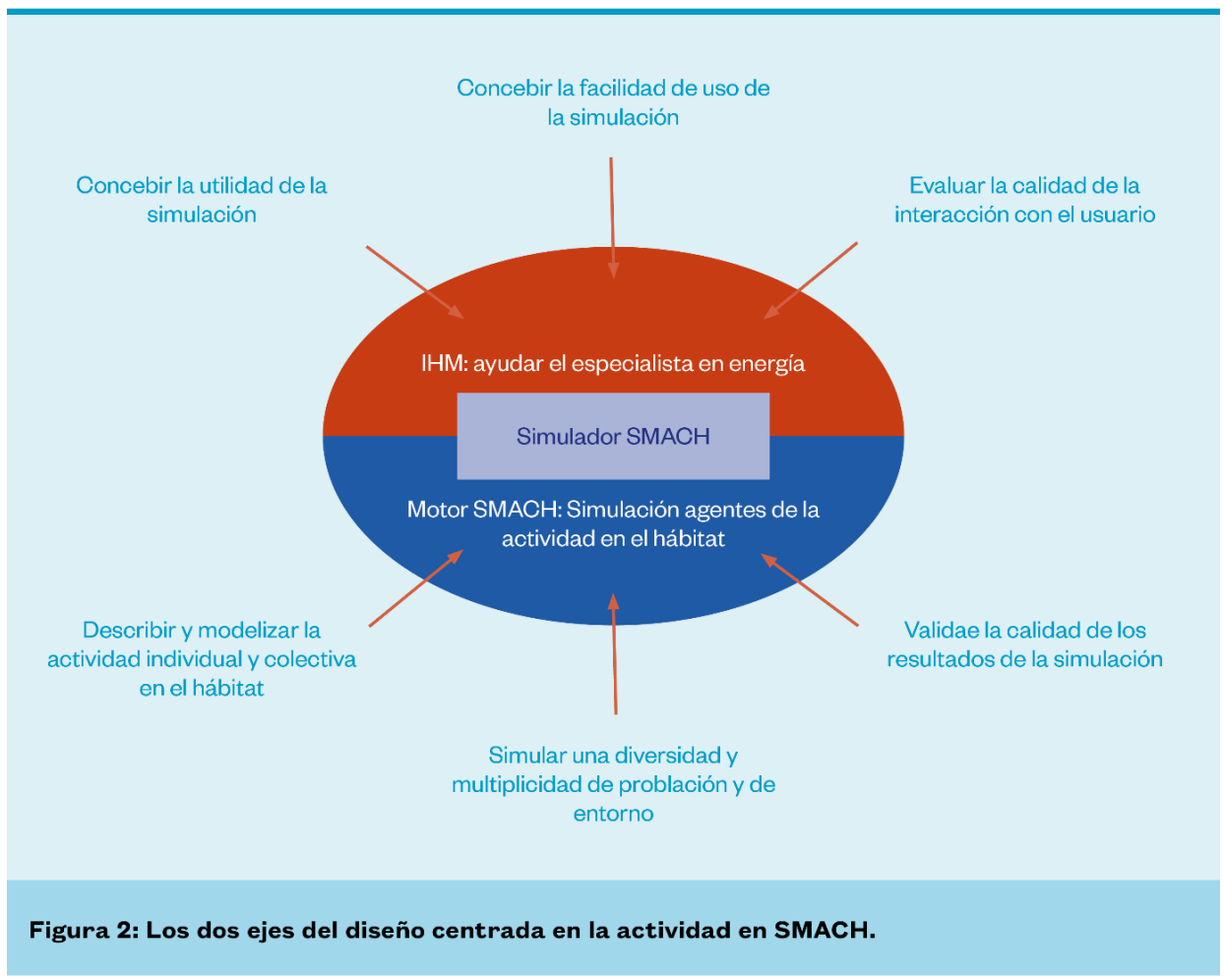

Figura 2: Los dos ejes del diseño centrada en la actividad em SMACH

12 Como lo muestra el esquema, debemos distinguir dos partes diferenciadas en una plataforma de simulación:

- La IHM, corresponde a la interacción con el usuario. Se la concibe en una perspectiva de ayuda al usuario, es decir, definiendo las funcionalidades útiles, un diálogo fácil de utilizar. Las IHM fueron sistemáticamente evaluadas en situación real o realista cada vez que eran orientadas hacia los expertos en energía o a la población en general;

- El motor de actividad y de consumo SMACH corresponde a la simulación basada en agentes de la actividad humana y de los consumos de energía. Está pensado para permitir una simulación lo más verosímil posible a fin de proponer resultados de cálculo pertinentes para las necesidades del experto en energía. La validación consiste entonces en calificar la simulación en relación con el fenómeno real. Este texto tratará principalmente acerca del diseño del motor de actividad.

13 La investigación tecnológica SMACH se desarrolló a lo largo de 12 años, en los que es posible distinguir 3 etapas muy estructurantes, como lo mostramos en la figura 3. 
Figura 3: Las etapas del programa de investigación tecnológico SMACH

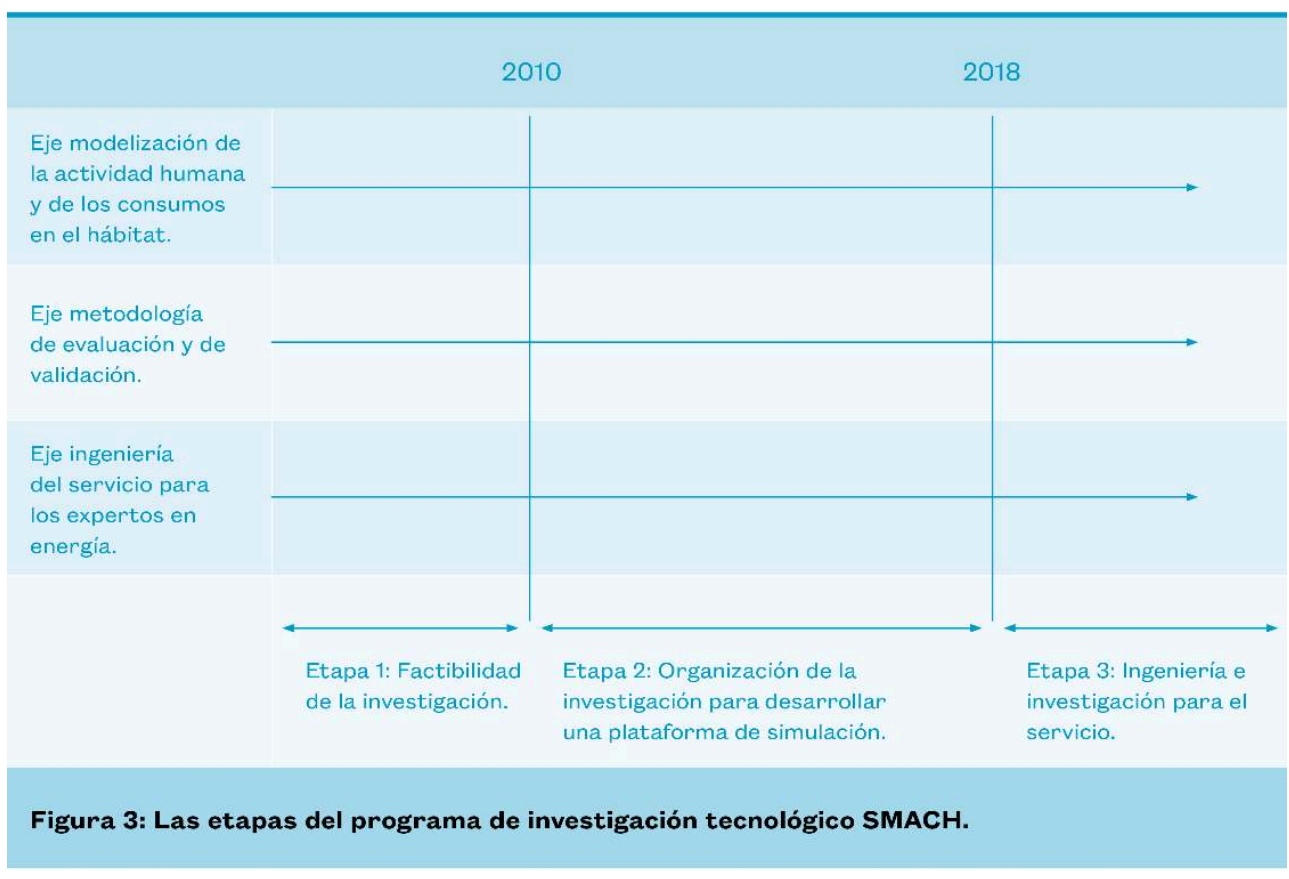

Figura 3: Las etapas del programa de investigación tecnológico SMACH

- La primera etapa corresponde a lo que se puede llamar una prueba de concepto, una etapa de factibilidad en la que tratamos definir si es posible simular una actividad doméstica, respetando las grandes características genéricas de la actividad humana.

- La etapa 2 es la que estructura la investigación en diferentes ejes de investigación necesarios para el desarrollo de la plataforma de simulación para que sea útil y utilizable.

- El éxito de las etapas precedentes culmina en el diseño de una plataforma de simulación y desemboca, en la etapa 3, en el desarrollo de una ingeniería de servicio y en la renovación de los interrogantes de investigación a raíz de las múltiples demandas de los expertos en energía.

Esta presentación de la organización del PIT SMACH a lo largo de varios años no debe ocultar el hecho de que se trata de un desenvolvimiento sin determinismo: en todo momento de la investigación, y por distintos motivos (científicos, estratégicos, presupuestarios, reglamentarios, ...), puede ser interrumpido o tomar caminos diferentes. En los capítulos siguientes, retomamos las distintas etapas de esta investigación tecnológica para observar las principales dinámicas de su construcción/ emergencia y de su organización. La investigación tecnológica, tal como la abordamos, corresponde a la construcción de un "curso de la investigación" que se elabora articulando diferentes investigaciones empíricas y técnicas.

\section{Etapa 1: Evaluar la factibilidad de la simulación de la actividad humana}

La pregunta que se plantea en esta etapa inicial de la investigación SMACH es la siguiente: ¿Es técnicamente posible y razonable, sin deformar demasiado un fenómeno 
real como la vida cotidiana en el hábitat, simular "in silico" una actividad humana? Consideramos entonces que el enfoque multiagente era interesante:

- Los Sistemas Multiagentes presentan características que los hacen aptos para simular en parte un comportamiento humano debido a la autonomía de los agentes (Ferber, 1995). La modelización multiagente hace posible simulaciones dinámicas en razón de las interacciones entre agentes o de la interacción de los agentes con su entorno.

- Es posible simular ciertas dimensiones de la experiencia humana apoyándose en una modelización informática que corresponde a una reducción de procesos sociales (Lefèvre, 2016). Nuestra idea entonces es que agentes autónomos representan a actores humanos que organizan sus actividades individuales y colectivas en el hábitat.

Sobre dichas bases, organizamos nuestro compromiso en la investigación tecnológica SMACH alrededor de dos preguntas (ver figura 4): 1) ¿Qué modelización de la actividad humana para respetar un nivel suficiente de semejanza con la actividad humana? 2) ¿Cómo confrontar el fenómeno simulado con el fenómeno real para asegurar ese nivel suficiente de semejanza?

Figura 4 : Un compromisso estruturado en torno a la cuestión de la simulación de la actividad y de su validación

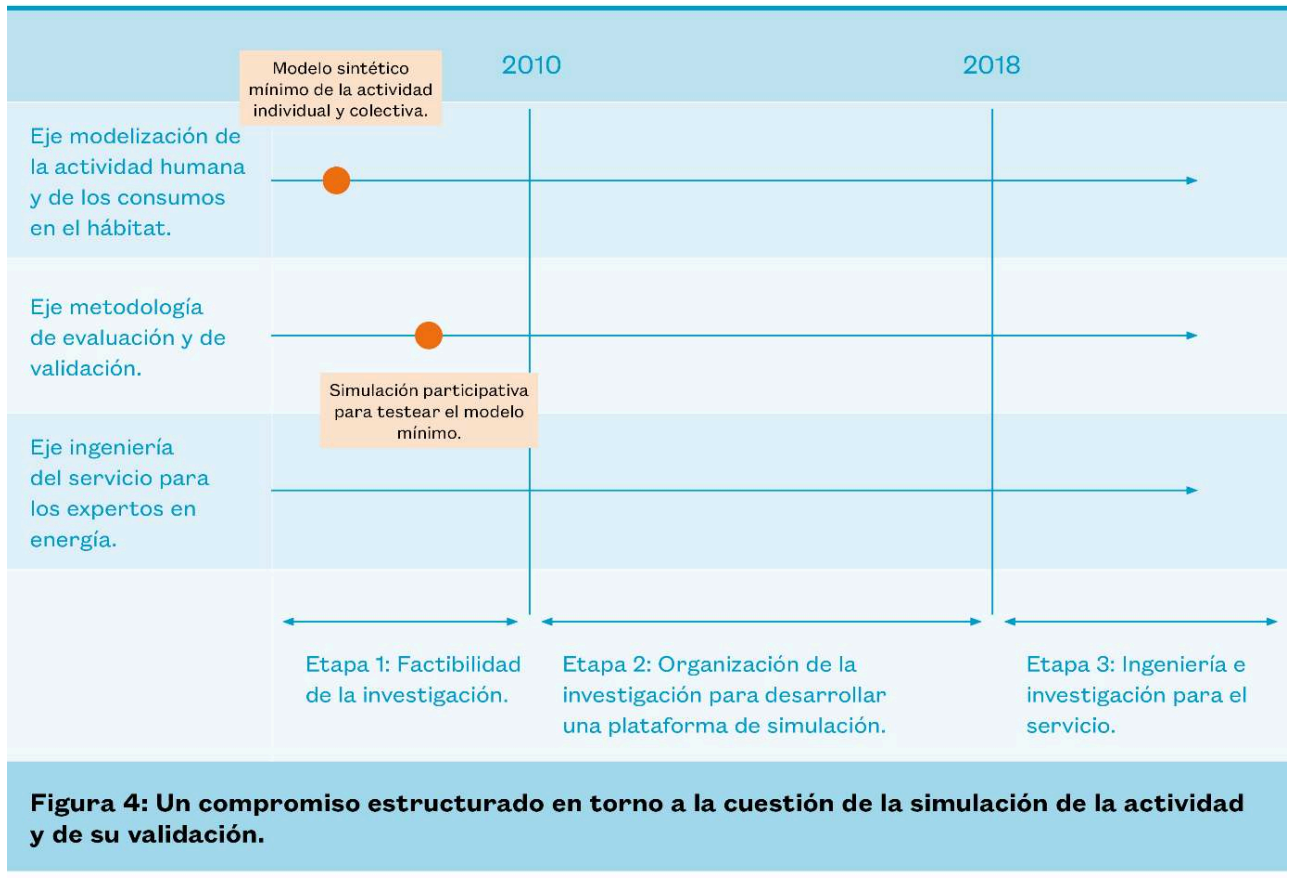

Figura 4 : Un compromisso estruturado en torno a la cuestión de la simulación de la actividad y de su validación

17 Esas dos preguntas van a organizar esta etapa de factibilidad y definir sus límites. Desarrollamos a continuación esos dos puntos.

\subsection{Un modelo sintético para una reducción aceptable in silico de la actividad humana}

18 Nuestro compromiso en esta investigación estuvo guiado en un primer momento por una hipótesis ontológica. Consideramos que el mundo de los seres vivos (el 
conocimiento empírico de la actividad humana) no debe ser confundido con la simulación de los seres vivos. Las lógicas que creamos en la simulación no tienen sentido más que para ese mundo computacional. La modelización multiagente de la actividad tiene sólo por función la de producir una imitación aceptable de la actividad humana. La actividad humana es entonces una fuente de inspiración y no atribuimos a la simulación que realizamos ninguna realidad cognitiva, emocional, cultural, etc. Nuestro objetivo es entonces alcanzar un nivel satisfactorio de imitación, definir la reducción del fenómeno que sea pertinente, que no lo desnaturalice: hablaremos entonces de verosimilitud de la simulación.

Durante esta primera etapa, realizamos algunos ensayos infructuosos de modelización multiagente como por ejemplo, modelizar los procesos cognitivos de decisión de diferentes actores. Finalmente, nos focalizamos en el nivel organizacional de la actividad humana en el hábitat: las dinámicas individuales y colectivas de los agentes hacen que emerja una organización de vida cotidiana en el hogar. Ese primer tipo de modelización corresponde a un modelo sintético, es decir, un modelo formal multiagente de la actividad humana. Este ensayo de modelización fue considerado satisfactorio puesto que se apoya sobre tres hipótesis teóricas de la enacción y del curso de acción:

- La primera hipótesis tiene que ver con la autonomía de los sistemas vivos en la corriente teórica de la enacción (Varela, 1989; Winograd, \& Flores, 1986). Todo sistema vivo constituye un sistema autónomo que debe mantener su equilibrio en la dinámica de sus interacciones con el entorno.

- La segunda hipótesis (Theureau, 2006; Varela, 1989) es relativa al actor humano que determina su actividad en función de su historia y del entorno en el que se encuentra. Es quien transforma su entorno y se transforma en la dinámica de sus interacciones. Esta interacción es asimétrica, pues es el actor el que define lo que es significativo/pertinente para él en la situación.

- Por último, con la tercera hipótesis (Theureau, 2006), consideramos la actividad colectiva como una totalidad cuya organización es constantemente cuestionada por las actividades individuales y constantemente reconstruida por esas mismas actividades individuales. El colectivo no es pues una unidad autónoma, pues corresponde a una "totalidad" constantemente "destotalizada" y "retotalizada" por la actividad de sus componentes.

En el siguiente ejemplo, generado automáticamente, ilustramos esta incorporación de los principios teóricos en la estructuración de la simulación. En la figura 5, cada línea horizontal corresponde a un agente y cada color a una acción. Por ejemplo, el agenteLuc (el padre) concatena un conjunto de acciones destinadas a ocuparse del agente-Tom (su hijo de corta edad), a ponerse de acuerdo con el agente-Eve (su pareja) y a organizar luego su velada (comida y televisión). 
Figura 5: Concatenación y coordinación de acciones individuales y colectivas en el modelo inicial SMACH

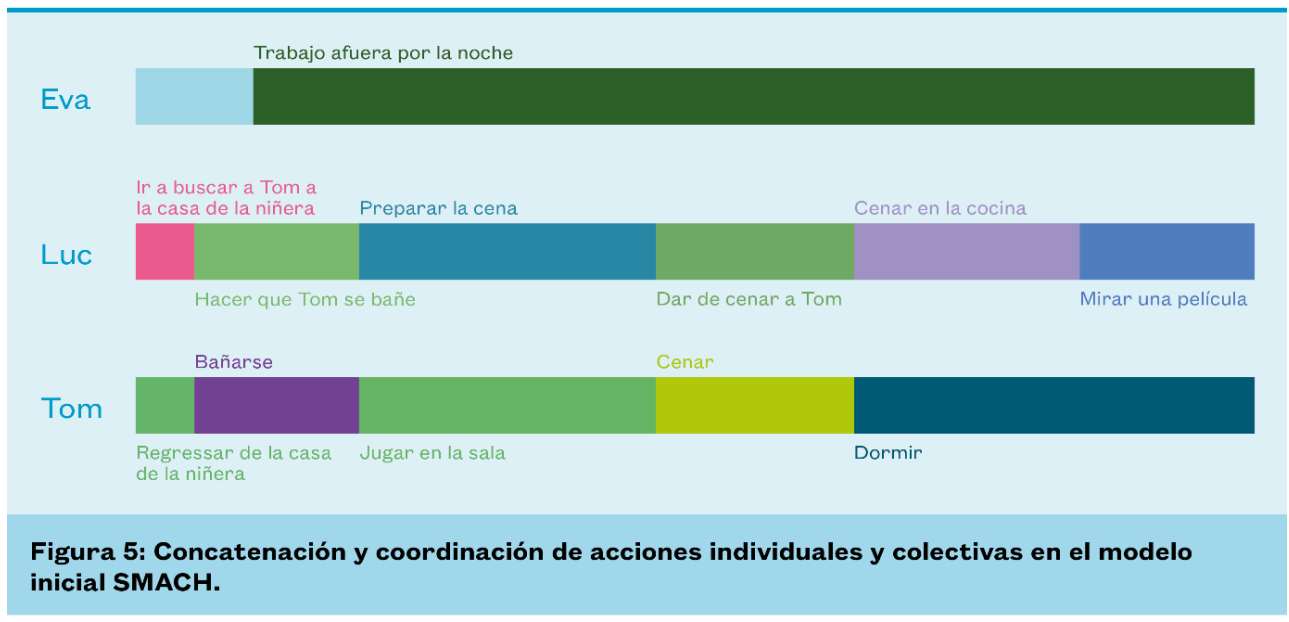

Figura 5: Concatenación y coordinación de acciones individuales y colectivas en el modelo inicial $\mathrm{SMACH}$

Consideramos que esta modelización sintética de la actividad humana produce una simulación verosímil de la actividad humana [5], dado que se estructura alrededor de los siguientes ejes:

- Se trata de simular una dinámica de actividad, es decir, una realización de acciones que evolucionará en función del entorno y de los agentes. Las acciones e interacciones no están predeterminadas: los agentes tiene un cierto nivel de autonomía [ $\left.{ }^{6}\right]$.

- La actividad de cada agente está vinculada al punto de vista específico que tiene de la situación (la habitación en la cual se encuentra, las acciones realizadas y las posibles de realizar).

- La actividad colectiva de los agentes es posible pues se comunican para coordinarse, ya sea para comer juntos, ocuparse de un niño por la mañana, etc.

Nuestro modelo sintético, en esta etapa, es muy limitado: sólo permite simulaciones de algunas horas, no dispone de un cálculo que integre el edificio, los equipamientos, la meteorología, etc. No obstante, muestra que es posible realizar una simulación verosímil de la actividad de la vida cotidiana en el hábitat. Este modelo sintético multiagente corresponde a una "reducción aceptable in silico de la actividad humana", pues está estructurado alrededor de algunos grandes principios teóricos de la actividad humana y respeta dinámicas de la vida cotidiana descriptas en nuestras investigaciones empíricas sobre la actividad humana en el hábitat (Guibourdenche et al., 2007; Haué, 2003).

Este modelo sintético para el diseño es, retomando los términos de Vinck y Laureillard (1995) un objeto intermediario de diseño que da cuenta de la construcción colectiva en el diseño. Es un mediador que materializa las intenciones de los diseñadores y que supera sus respectivos aportes. Pero ese modelo sintético es más que eso, puesto que corresponde a lo que Haué (2003) llama un modelo pivote: se construye articulando la descripción de la actividad humana con la modelización informática. Encontramos en este tema del diseño multiagente modelos similares (Guyot, 2006; Le Page, 2017) entre los que se distinguen tres tipos. El modelo del experto en el campo, el modelo intermediario, formal, que es el modelo de diseño y finalmente, el modelo técnico, realizado por el informático. Esta descripción general es parecida a nuestra práctica de 
modelización aunque no es muy precisa sobre lo que se llama "modelo de negocios". En lo que nos concierne, el modelo de la actividad humana se apoya en nuestro diagnóstico/pronóstico y el modelo sintético lo integra en el marco de las lógicas formales de la modelización agentes. Retomamos a continuación el esquema de Haué (2003) sobre el que introdujimos especificaciones de manera de hacerlo corresponder con nuestra práctica.

Figura 6: El modelo sintético como modelo pivote del diseño

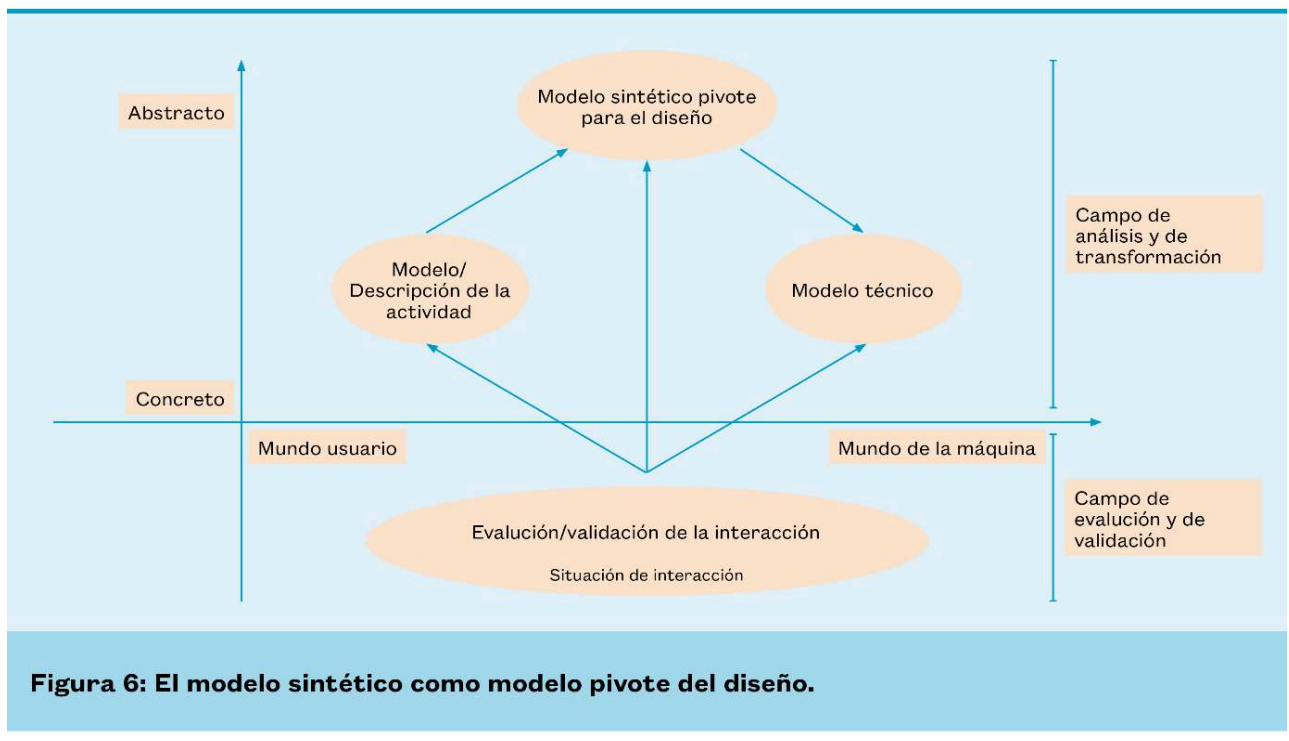

Figura 6: El modelo sintético como modelo pivote del diseño

El modelo sintético tal como lo proponemos es un resultado de modelización significativo para la investigación tecnológica: concreta lo que puede ser una "reducción aceptable in silico de la actividad humana", aporta una respuesta a nuestra necesidad de simular la actividad humana. Un resultado de modelización significativo tiene un rol rector en las acciones futuras en la plataforma multiagente: las opciones que se escojan serán determinantes para la continuidad de los trabajos.

Este modelo sintético es una base mínima de modelización estructurada alrededor de grandes principios teóricos y prácticos de la actividad y de la simulación basada en agentes: es un modelo limitado, un bosquejo del modelo futuro. Si es validado, ya no podrá ser cuestionado.

\subsection{La simulación participativa para evaluar el modelo sintético limitado de la actividad humana}

En el marco de esta etapa de factibilidad, es esencial cerciorarse del potencial del modelo sintético para simular una actividad humana, verificar la facilidad de su implementación, determinar su capacidad de evolución. La cues tión que se plantea entonces en esta fase, es cómo construir un observatorio lo suficientemente realista como para poder confrontar el fenómeno simulado con el fenómeno real.

La simulación participativa interactiva (Le Page, 2017) es un recurso metodológico que se implementa con frecuencia en los enfoques agentes. La simulación participativa coloca al actor humano en el centro del sistema (Guyot, 2006) al implicarlo en la 
situación simulada. Un actor humano, con la simulación participativa de SMACH, puede situarse en un hábitat simulado y realizar acciones de la vida cotidiana: el actor humano, por intermedio de su avatar, realiza acciones (levantarse, lavarse, etc.), se coordina con los otros actores, etc.

Para evaluar la verosimilitud de la simulación, se hace una experimentación a fin de asegurarse de que esa modelización sintética mínima sea un punto de partida adaptado para dar cuenta de la actividad humana en el hábitat. La experimentación se elabora sobre las bases metodológicas de la evaluación ergonómica con el objetivo de aproximarse lo más posible a una situación futura (Pinsky \& Theureau, 1984). Para eso, simulamos una situación de vivienda compartida, invitamos a tres personas en participar de la experimentación y definimos dos personajes virtuales que interactúan en el escenario simulado. La experimentación se compone entonces de tres actores humanos y dos agentes automáticos. Las modalidades de la experimentación son las siguientes:

- Los actores humanos ignoran el rol que desempeñan los otros actores humanos. Tampoco saben qué personajes son desempeñados por los agentes automáticos.

- La experimentación se desarrolla en varias fases para representar varias jornadas.

- La experimentación se filma y al final, se realiza una entrevista.

El resultado más importante de la experimentación tiene que ver con la validación del modelo sintético de la simulación. Los actores humanos lograron crear o recrear momentos de vida realistas. Por ejemplo, un actor humano que justamente compartía vivienda, nos dijo que había reproducido comportamientos que tenía en su casa. También observamos comportamientos colectivos significativos y no anticipados: de una fase a otra de la experimentación, algunos actores humanos construyen una experiencia y adaptan sus acciones en consecuencia. Por ejemplo, una persona no quiso lavar los platos puesto que lo había hecho en las dos fases anteriores. Además, los agentes automáticos sólo fueron identificados tardíamente. La capacidad de los actores humanos para "vivir" esos momentos e interactuar "naturalmente" con agentes automáticos muestra el buen nivel de verosimilitud del modelo.

Esta experimentación mostró que la simulación participativa es una herramienta metodológica posible para calificar el nivel de verosimilitud de la simulación de una actividad humana. Al crear una situación de utilización realista, hemos creado un objeto intermediario de diseño que, esta vez, tiene una finalidad metodológica relativa a la experiencia humana. Esta construcción corresponde a un resultado metodológico significativo para la investigación tecnológica que trata sobre la adecuación de la simulación a la actividad humana. Dicha elección de diseño metodológico "diseño participativo orientado a la validación" corresponde entonces a una instancia de test del modelo sintético. Podemos retomar el esquema de Haué (2003) y completarlo (ver figura 7), en su parte inferior, para explicar dicha construcción metodológica. La elección del diseño metodológico es, esta vez, el resultado de un proceso invertido respecto del diseño: las consideraciones técnicas (modelo sintético, IHM para la interacción de simulación participativa...) están al servicio de la situación pivote de experimentación. 
Figura 7: El diseño participativo como metodologia pivote de la evaluación/validación de la simulación

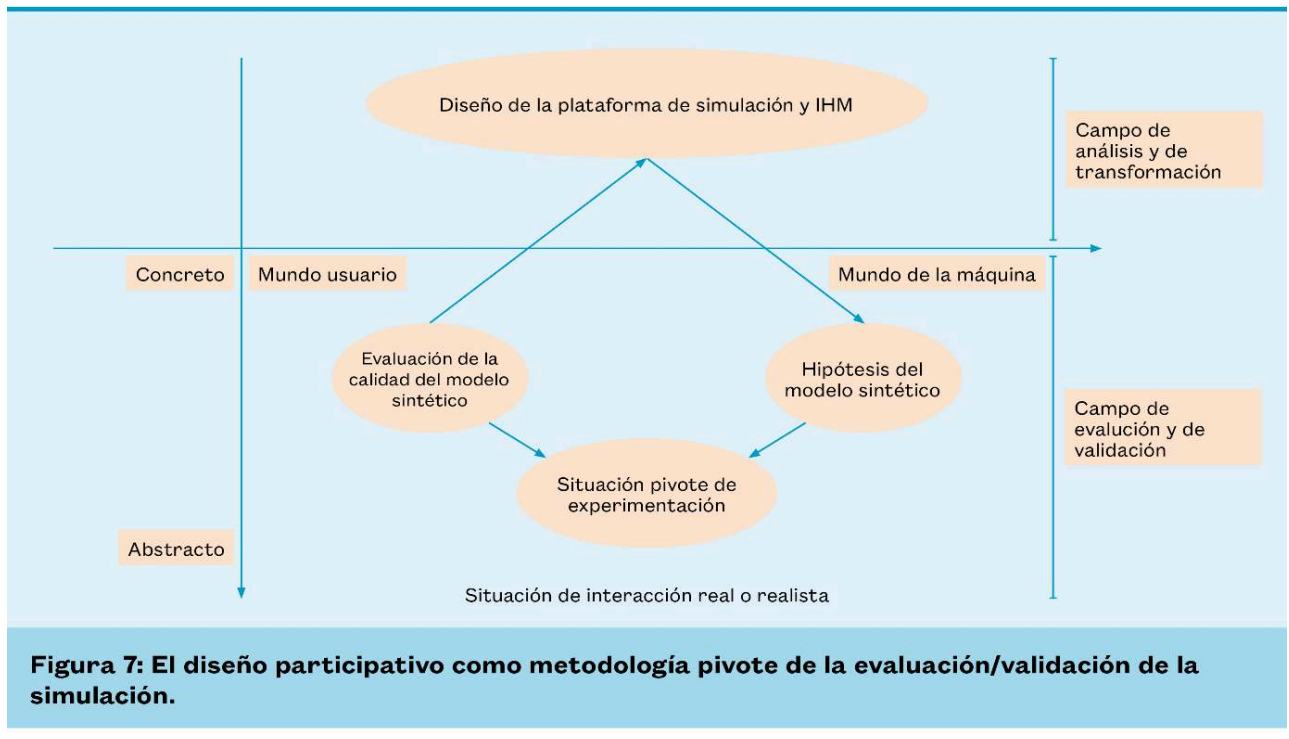

Figura 7: El diseño participativo como metodologia pivote de la evaluación/validación de la simulación

Esta experimentación de simulación participativa corresponde al final de la primera etapa de la investigación tecnológica SMACH. El diseño de un primer modelo de actividad en el hábitat, muy simplificado, y su confrontación con actores humanos permitieron ir más allá de una intuición: la simulación multiagente de la actividad humana en el hábitat se torna una posibilidad real. Estos primeros trabajos demostraron la factibilidad de esa orientación de investigación. Mostraron asimismo la relación estructural existente en dicho PIT: 1) entre la actividad humana y el diseño de un modelo sintético, 2) entre esa modelización y su confrontación con una situación experimental de simulación participativa (Haradji, Poizat, \& Sempé, 2012). Los resultados positivos de esta etapa son determinantes: abren una nueva etapa de la investigación tecnológica.

\section{Etapa 2 : el desarrollo del programa de investigación tecnológico para simular a escala de un hogar y de una población}

Las conclusiones positivas de la etapa de factibilidad hicieron posible el desarrollo del programa de investigación tecnológico SMACH. En esta segunda etapa, dos momentos fueron importantes: 1) concebir la simulación de un hogar y su validación, 2) concebir la simulación de una población y su validación. Desarrollaremos a continuación esos dos momentos del PIT SMACH.

\subsection{El modelo sintético avanzado a escala de un hogar y su validación}

El modelo sintético limitado, desarrollado durante la etapa 1 de factibilidad, demostró ser prometedor. Sirvió como punto de partida para la realización de un modelo más 
completo de la actividad humana en su ecosistema. En un primer momento, quisimos simular la continuidad de la actividad humana durante períodos prolongados. Para comprender cómo se organiza la actividad humana día a día, partimos de nuestros estudios empíricos del curso de acción relativos a la vida cotidiana en el hábitat (Guibourdenche et al., 2007). De este modo, se completó el modelo sintético inicial para integrar hábitos de vida durante el funcionamiento de la simulación (Haradji et al., 2018). En un segundo momento, quisimos modelizar el entorno del hábitat para crear una situación de interacción lo más verosímil posible para los agentes. Integramos entonces modelos técnicos del sistema térmico del edificio (Plessis, Amouroux, \& Haradji, 2014), de meteorología, de equipamientos eléctricos (por ej., refrigerador, calefacción...), y ofertas tarifarias. El modelo multiagente resultante es un modelo sintético avanzado que corresponde a una "reducción aceptable in silico de un hogar en su ecosistema" (ver figura 8): permite simular la actividad de un hogar y su consumo de energía.

Figura 8: El desarrollo del modelo sintético avazado para un hogar y su validación

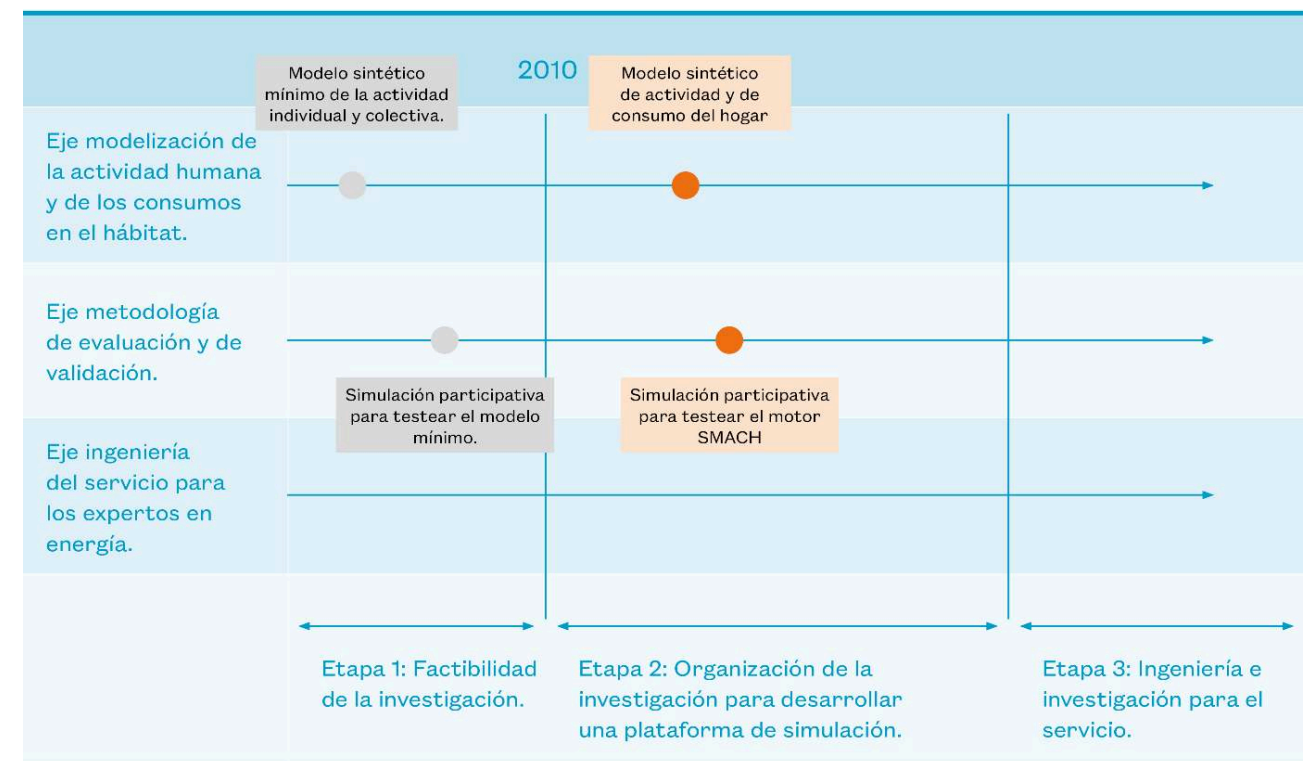

Figura 8: El desarrollo del modelo sintético avanzado para un hogar y su validación.

Figura 8: El desarrollo del modelo sintético avazado para un hogar y su validación

Este modelo sintético avanzado es un modelo formal multiagente que da cuenta de la complejidad de las interacciones en el hábitat y que respeta las hipótesis teóricas de la enacción y del curso de acción (ver capítulo anterior). De esta manera, podemos entonces distinguir en el esquema multiagente de la figura 9:

- La autonomía y la dinámica de la actividad individual del agente. La actividad individual es llevada a cabo por un agente (un individuo [7]) que realiza tareas de manera autónoma. En el transcurso de la simulación, emergen hábitos que reciben la influencia de modos de vida relacionados con la eficiencia energética, el confort, el presupuesto.

- La construcción de la actividad colectiva de un agente con otros agentes. La actividad colectiva se construye en la interacción entre agentes (los individuos) que juntos realizan tareas y forman para esa ocasión grupos (o colectivos) efímeros, compuestos por todos o parte de los miembros del hogar (adultos, niños, parientes...). 
- El acoplamiento asimétrico del agente con su entorno. El acoplamiento estructural con el entorno es el resultado de los agentes (los individuos), comprometidos con sus actividades individuales y colectivas, que están en interacción con un entorno compuesto de aparatos (calefacción, termo-tanque, refrigerador, etc.) y de habitaciones que constituyen una vivienda con un cierto nivel de aislación. Además, el proveedor propone ofertas tarifarias.

Figura 9: El modelo de la organización multi-nivel de la actividad humana (Huraux, Sabouret, \& Haradji, 2015)

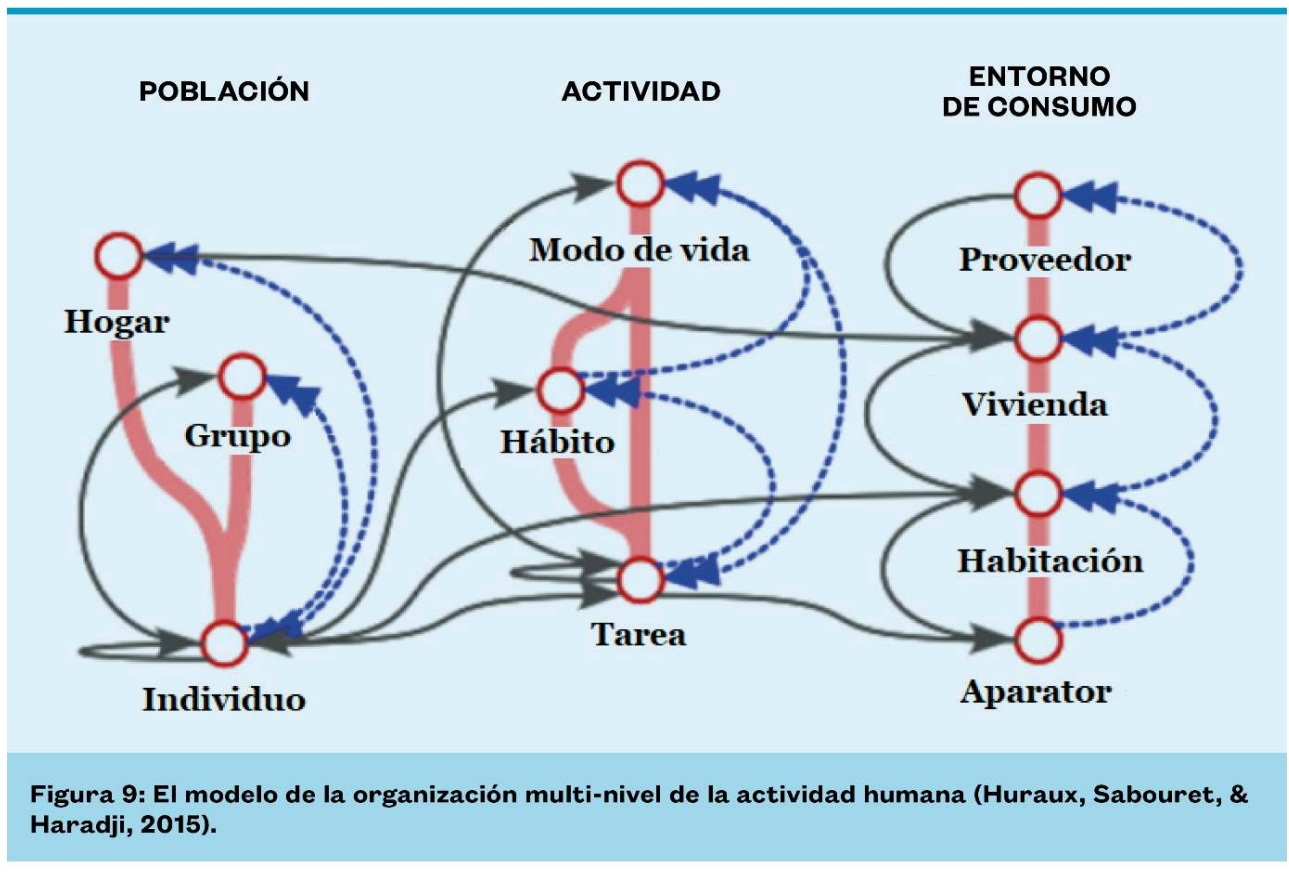

Figura 9: El modelo de la organización multi-nivel de la actividad humana (Huraux, Sabouret, \& Haradji, 2015)

Como dijimos antes, se trata de un modelo pivote de diseño, un modelo sintético avanzado, que dio lugar a un modelo informático.

Para la validación del modelo, retomamos el principio metodológico de la simulación participativa y lo implementamos en situaciones naturales involucrando a familias verdaderas. Respetamos tres principios para mejorar el carácter "natural" de la experimentación:

- Utilizar una situación real de referencia sobre los consumos de electricidad que permita la comparación con la simulación. EDF (Électricité de France) realiza diferentes experimentaciones en situación real. La experimentación elegida fue "Una Bretaña [ $\left.{ }^{8}\right]$ adelantada", puesto que se disponía de datos cuantitativos sobre el consumo de alrededor de 500 familias.

- Confrontar a los miembros de una familia con la simulación de su vida cotidiana. Para evaluar el realismo de la simulación, involucramos a familias por medio de la simulación participativa y las confrontamos con la simulación de su vida cotidiana.

- Articular las validaciones cualitativa y cuantitativa. Quisimos validar la simulación desde una perspectiva cualitativa (la actividad en el hábitat), pero también desde una perspectiva cuantitativa (las curvas de consumo de energía).

Para crear condiciones satisfactorias de experimentación: 1) desarrollamos una interface de simulación participativa que haga posible que un actor humano manipule 
un avatar y pueda así modificar el curso de la simulación (figura 10);2) realizamos entrevistas previas a diez familias provenientes de la experimentación "Una Bretaña adelantada" para estar en condiciones de realizar una simulación de una semana para cada una de las diez familias; 3) confrontamos a un actor humano de la familia con su dinámica de vida cotidiana simulada. Dicho actor describe y comenta esta actividad "in silico" en relación con su actividad real.

Figura 10: Diseño de un entorno realista para la simulación participativa

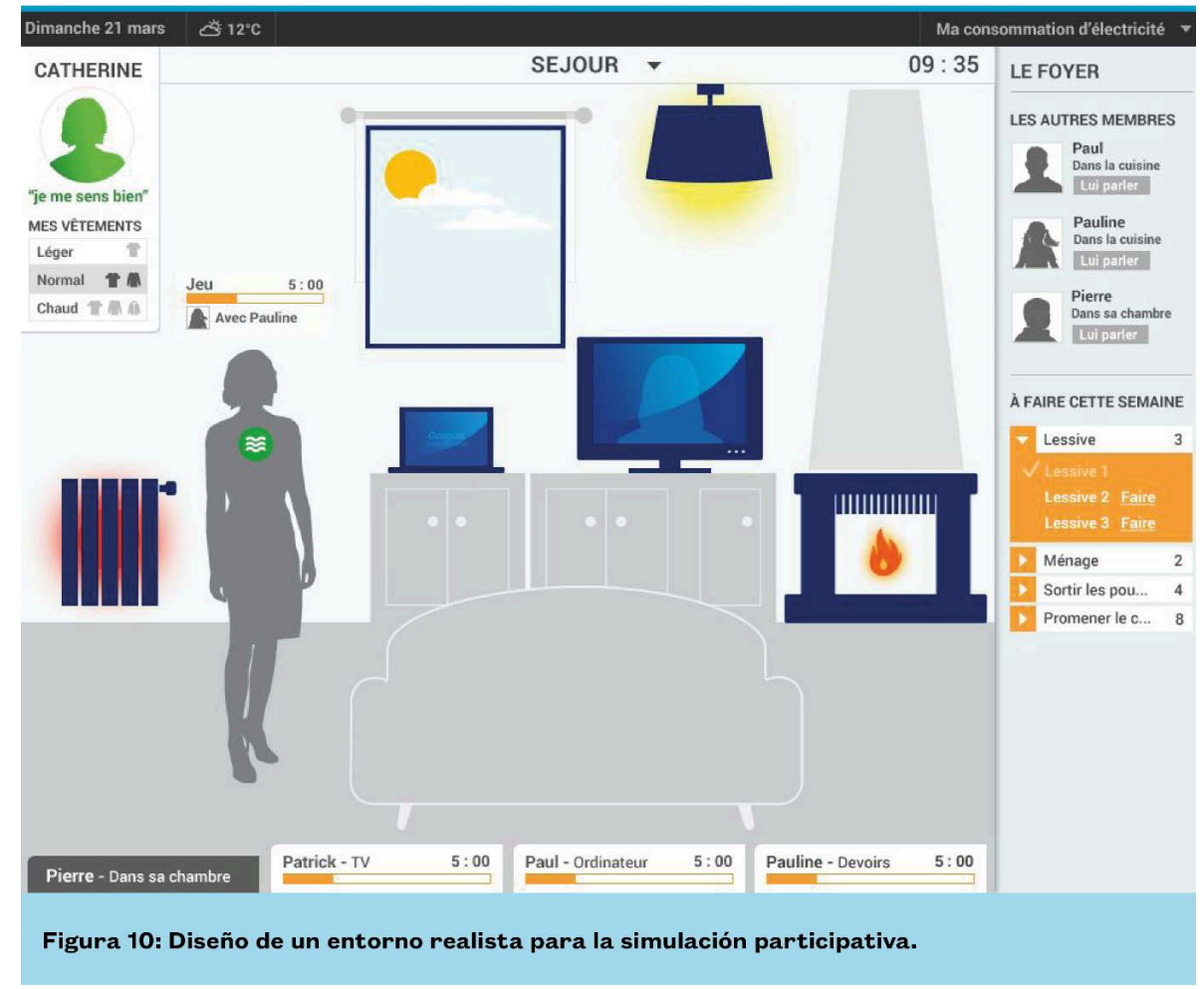

Figura 10: Diseño de un entorno realista para la simulación participativa

Los resultados de la experimentación son concluyentes en lo que respecta a la dinámica de vida cotidiana en el hábitat. Ninguno de los mecanismos de base de la simulación (autonomía de los agentes, dinámica individual y colectiva, acoplamiento con el entorno) fue cuestionado. Una validación de los consumos completó esa validación cualitativa del modelo sintético avanzado. Para eso comparamos las curvas de carga simuladas de esas familias con sus consumos reales registrados en el marco de la experimentación "Una Bretaña adelantada” (figura 11). 
Figura 11: Ejemplo de comparación de la curva de carga simulada (en azul) com las curvas de carga reales de la família (en rojo)

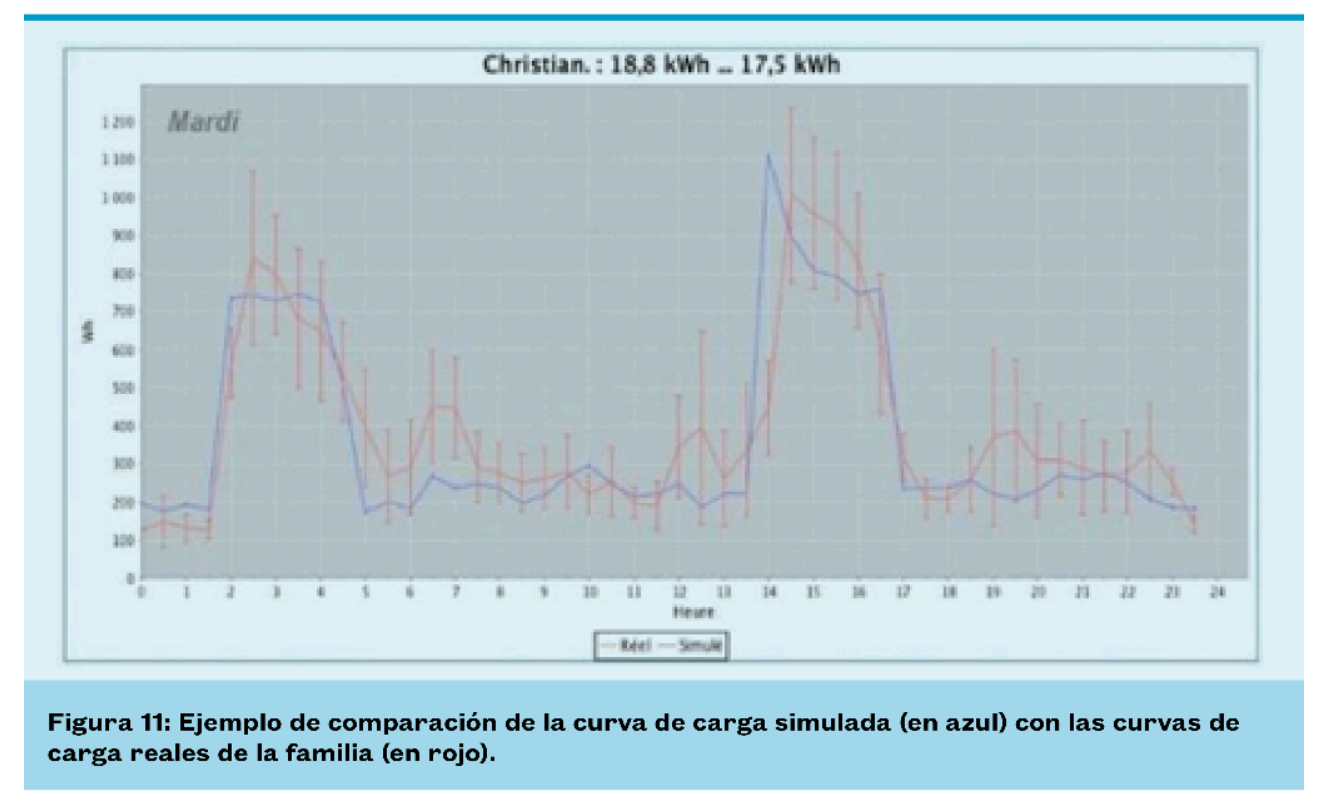

Figura 11: Ejemplo de comparación de la curva de carga simulada (en azul) com las curvas de carga reales de la família (en rojo)

Los resultados de la evaluación del motor SMACH demostraron ser positivos (Haradji et al., 2018): a escala de un hogar, son verosímiles en cuanto a la actividad humana (validación cualitativa) y los consumos de energía son comparables a los consumos reales (validación cuantitativa).

El modelo sintético avanzado queda validado: permite simular la actividad de un hogar y calcular los consumos de energía que de ella derivan. Se hace posible hacer cálculos iniciales de eficiencia energética (por ejemplo, calcular el impacto de una nueva tarifa sobre el consumo de energía).

\subsection{El modelo sintético avanzado a escale de una población y su validación}

41 La anticipación de los consumos energéticos rara vez se plantea a nivel de varios hogares. La simulación debe ser encarada a diferentes niveles de población para poder responder a la mayoría de los desafíos de negocio relativos a la energía en el hábitat (evaluar el impacto de una nueva oferta tarifaria, el impacto de una evolución reglamentaria, el impacto de eco-gestos, etc.). Pasar de simular algunos hogares a simular una población (ver figura 12) equivale a un nuevo desafío del PIT SMACH: cómo simular la multiplicidad y la diversidad de grupos humanos presentes en una población respetando el realismo individual de cada hogar. El cambio de escala no debe ser acompañado por el "aplastamiento" de las diversidades relativas a la actividad y a los consumos de los hogares: lo global no debe estar desconectado de lo local. 
Figura 12: El desarrollo del modelo sintético avanzado para una población y su validación

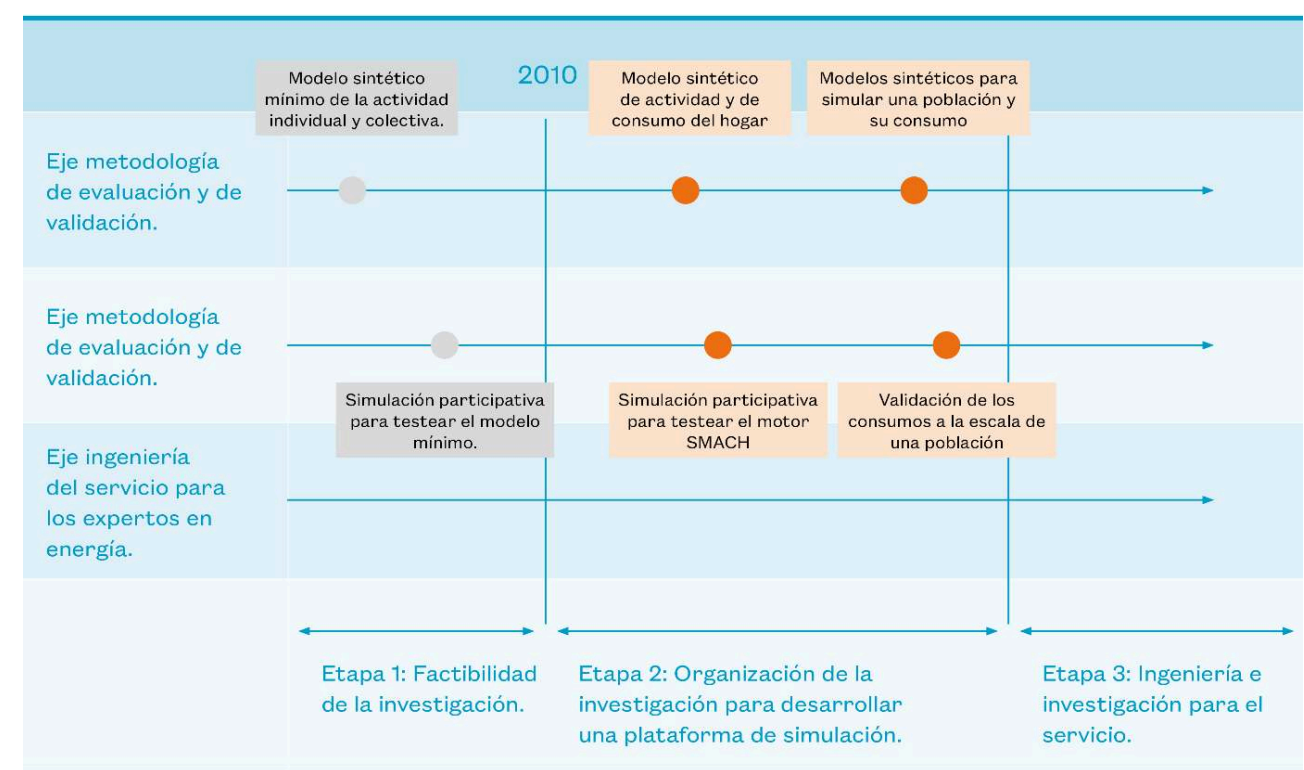

Figura 12: El desarrollo del modelo sintético avanzado para una población y su validación.

Figura 12: El desarrollo del modelo sintético avanzado para una población y su validación

Con este cambio de escala nos enfrentamos entonces a un problema epistemológico: no es posible observar y analizar la actividad de miles de hogares para definir, a gran escala, los modelos sintéticos de cada hogar de una población. Para superar esta limitación, separamos lo que pertenece al orden de la descripción estadística global de una población de hogares (población y distribución del tiempo) de lo que pertenece al orden de la actividad local de cada hogar en su hábitat. De esta manera, articulamos dos puntos de vista. El primero, estadístico, reposa sobre la encuesta de la distribución del tiempo realizada por el INSEE (Institut National de la Statistique et des Études Économiques) para la que cada uno de los 27000 hogares encuestados indicó, a lo largo de dos días, lo que hacía cada diez minutos. Esta base de datos socio-estadísticos presenta así la ventaja de: 1) reflejar un estado de las acciones realizadas en el hábitat, 2) describir una diversidad de hogares llevando a cabo una diversidad de acciones a escala de una población. Ese punto de vista global sobre las acciones posibles (las descripciones estadísticas para cada hogar) se articula entonces con el punto de vista local llevado adelante por el motor de actividad SMACH: los agentes "darán vida" pues a los datos estadísticos. Se adaptarán a su entorno térmico, tarifario, meteorológico y, con una cierta autonomía, construirán sus interacciones individuales y colectivas en el hábitat. Articulamos así el método estadístico, basado en modelos que apuntan a un realismo estadístico global, con el método agente, basado en modelos que apuntan a un realismo local en el hábitat (Reynaud, Haradji, Sempé, \& Sabouret, 2017). La simulación asociada a los datos estadísticos corresponde a un modelo sintético avanzado que produce una "descripción de la actividad a gran escala aceptable para anticipar consumos de energía".

Con ese modelo, se hace posible calcular consumos de energía a escala de un grupo de hogares, de una ciudad, de un país, etc. El procedimiento adoptado para la validación de los consumos a gran escala (Delenne, 2018) consistió en confrontar, en un primer 
tiempo, 1000 curvas de consumo simuladas por SMACH con valores de referencia del distribuidor Enedis (ver ejemplo en la figura 13). En un segundo tiempo, se compararon curvas de consumo simuladas con la suma de curvas de consumo reales de 10 hogares que habían participado en una experimentación en la ciudad francesa de Lyon.

Figura 13: Comparación de los perfiles semanales para los clientes en opción Base

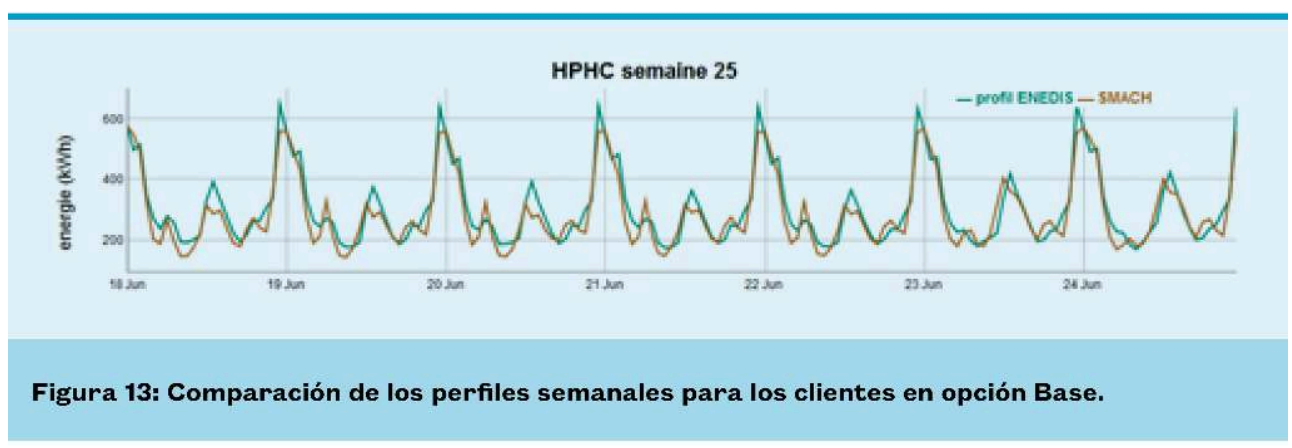

Figura 13: Comparación de los perfiles semanales para los clientes en opción Base

La comparación entre los consumos simulados y los consumos reales mostró que SMACH es lo suficientemente realista como para analizar el impacto de nuevos comportamientos, nuevos materiales o nuevas ofertas sobre los consumos de energía.

La realización de la simulación a gran escala y su validación cierran esta segunda etapa de la investigación tecnológica que corresponde al final del proceso de diseño de la plataforma de simulación. Es en este momento de la investigación tecnológica que la simulación de la actividad en el hábitat demostró ser muy útil para las profesiones relacionadas con la energía. La posibilidad de simular a escala de una población utilizando SMACH abrió el camino para una utilización importante de los diagramas de actividad y de las curvas de consumo. Se produjeron de este modo muchas decenas de miles de archivos de datos para responder a distintos escenarios de negocios como la presencia del vehículo eléctrico en el hábitat, el impacto de los gestos tendientes a la economía de energía a nivel del territorio, o incluso la evaluación del impacto de una oferta tarifaria sobre la curva de carga, etc.

\section{Etapa 3 : desarrollar el servicio y la investigación tecnológica asociada}

Las numerosas demandas de simulación demostraron que la plataforma SMACH respondía a una necesidad. Permitieron asimismo reconocer que nuestro compromiso con el PIT SMACH debía evolucionar. De una organización centrada en la factibilidad y el desarrollo de la simulación pasamos a ser una organización centrada en la producción de datos realistas para anticipar las situaciones futuras []. Es esa necesidad de los clientes SMACH la que dirige ahora la dinámica de los trabajos, ya sea que se trate de hacer evolucionar la plataforma (ingeniería del servicio) o de investigar sobre los modelos (ver figura 14). 
Figura 14: La etapa actual para e desarrollo de un servicio SMACH

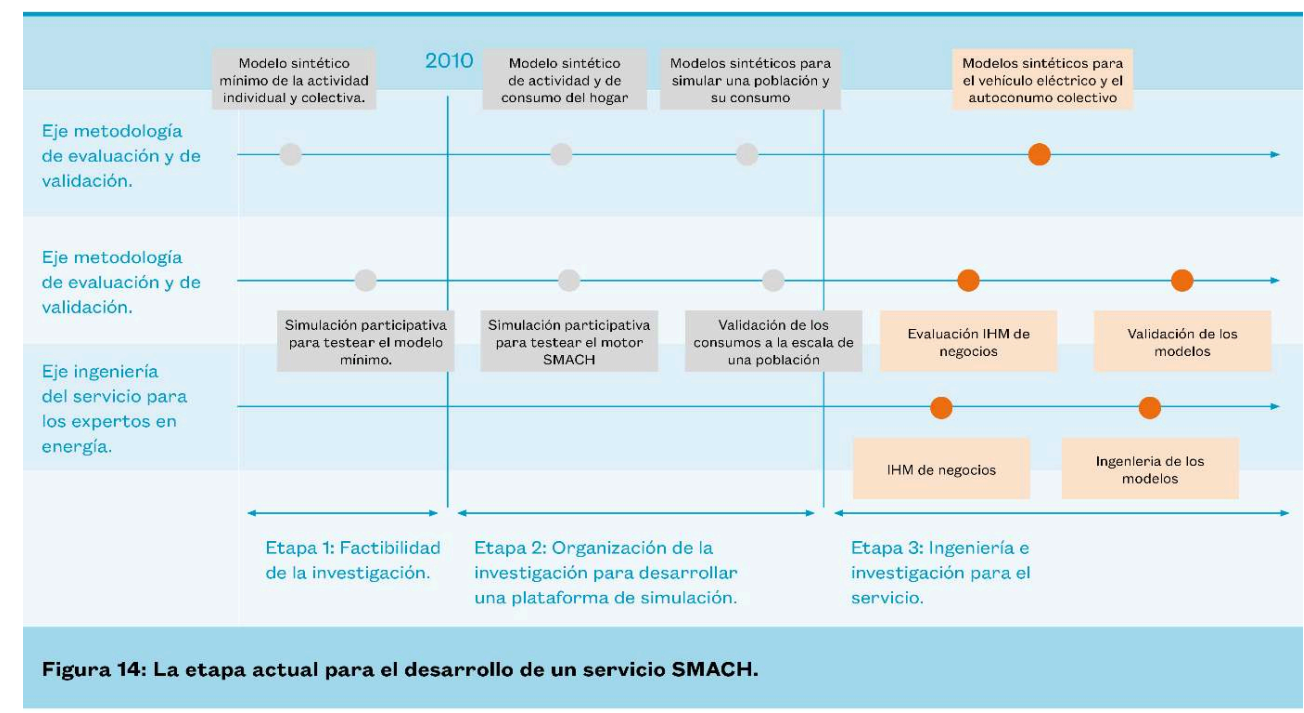

Figura 14: La etapa actual para e desarrollo de un servicio SMACH

Para responder a la demanda de datos realistas (diagrama de actividad y curvas de consumo), concebimos una IHM de negocios dedicada a los especialistas en energía (economistas, especialistas en instalaciones térmicas, en redes eléctricas...). Respetamos un proceso clásico de diseño centrado en la actividad humana (Haradji \& Faveaux, 2006) con: 1) una definición de la necesidad de los expertos a partir de un análisis de sus demandas y de una serie de entrevistas; 2) una especificación de la IHM y una evaluación sobre maqueta con los expertos (ver figura 15);3) un desarrollo informático de la IHM de negocios, de la arquitectura y de la base de datos. Asimismo, desarrollamos, en una perspectiva de servicio, nuevos modelos técnicos (modelos de renovación de edificios, de bombas de calor, de equipamientos eléctricos, etc.) que no necesitan atención (o poca) sobre el modelo de actividad humana. 
Figura 15: Desarrollo de una maqueta y de una aplicación de negócios para la utilización de SMACH

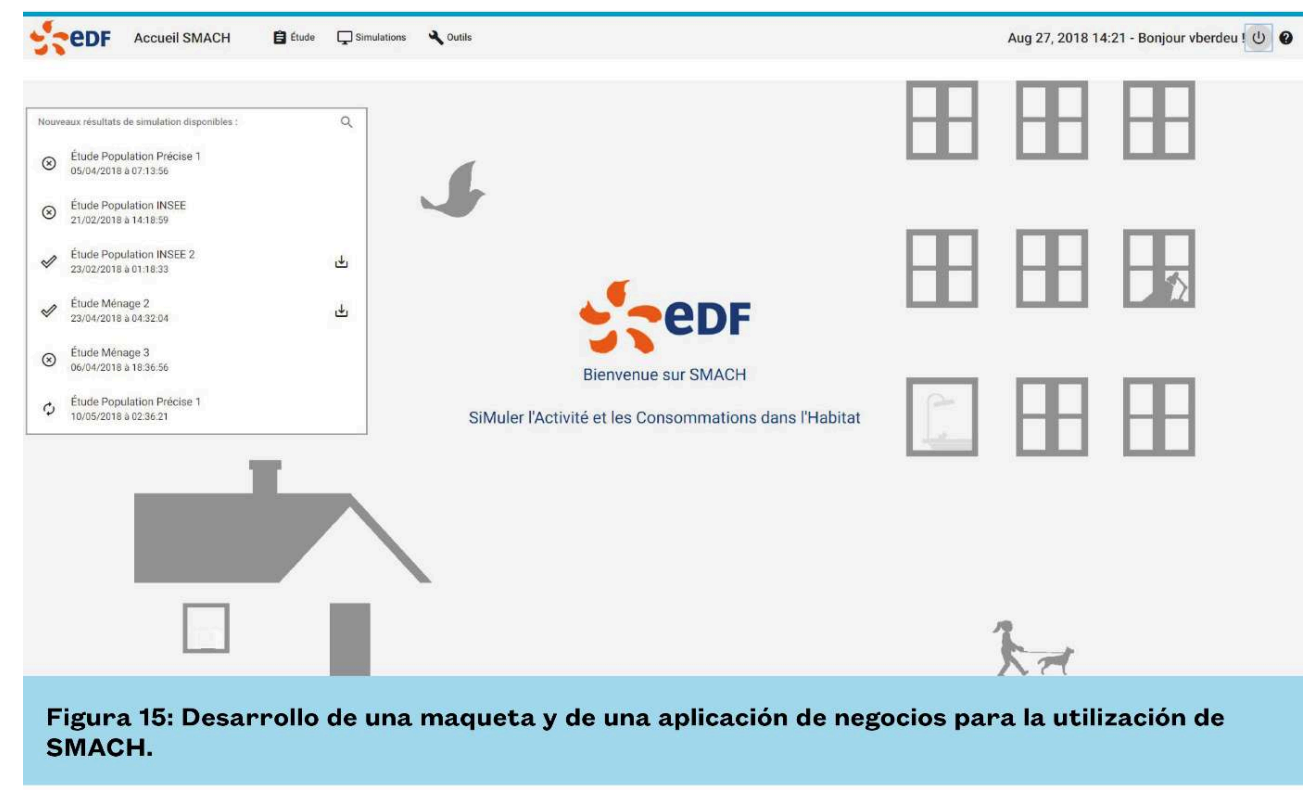

Figura 15: Desarrollo de una maqueta y de una aplicación de negócios para la utilización de SMACH

Debemos distinguir en cambio esta ingeniería (de la IHM, de los modelos técnicos) de las cuestiones de investigación que necesitan nuevas modelizaciones de la actividad humana. Por ejemplo, para calcular el consumo del vehículo eléctrico cuando se recarga en el hábitat, debemos modelizar los tipos de trayecto que hace el habitante con su vehículo, si lo recarga en su lugar de trabajo, en un centro comercial, en un espacio público... El nivel de carga de su vehículo determinará su necesidad de recarga en el hábitat. Ese trabajo, en vías de desarrollo, activa hipótesis que se apoyan en datos estadísticos sobre las actividades de las personas cuando se desplazan.

La extensión de SMACH hacia el autoconsumo colectivo abre un campo de investigación aún más amplio, pues presenta una nueva lógica de consumo de la electricidad. Con la plataforma actual, todos los hogares son independientes; pero con el autoconsumo colectivo, los agentes y en mayor medida los hogares, deberán comunicarse para compartir energía. Nos vemos entonces confrontados con un doble interrogante de investigación. En primer lugar, cómo hacer que la simulación SMACH evolucione técnicamente para integrar los mecanismos multiagentes del acto de compartir energía entre hogares o grupos de hogares (Albouys-Perrois, Sabouret, Haradji, Schumann, \& Inard, 2019). Se trata de una transformación profunda de la modelización agente como consecuencia de la creación de la noción de intercambio de energía que da lugar a un nivel de modelización superior al del hogar. Este interrogante de diseño obliga también a transformar el modelo de la actividad humana para que la decisión individual y colectiva se integre al acto de compartir entre hogares una producción intermitente de la energía (fotovoltaica, eólica). Nuestros trabajos anteriores en ergonomía no nos permiten hacerlo, pues ese tipo de experiencia humana es infrecuente. Actualmente, se está llevando a cabo una investigación en diseño y ergonomía (Bonnardot, Vial, Salembier, Prieur, \& Haradji, 2021) cuyo objetivo es documentar la actividad humana, individual y colectiva, en vistas a una situación futura que transforme el vínculo con la energía, las relaciones sociales y la dimensión económica de los hogares implicados en 
esa transición. Esta investigación permitirá entonces proponer hipótesis realistas de evolución de la actividad futura en un contexto de autoconsumo colectivo.

Nuestro compromiso en un servicio SMACH toma así dos direcciones complementarias. Los trabajos de ingeniería están dirigidos a responder a las demandas de expertos en energía. Nosotros los diferenciamos de las cuestiones de investigación tecnológica que promueven nuevas modelizaciones tanto a nivel de la dimensión técnica (los equipamientos) como a nivel de la formalización de la actividad humana (vehículo eléctrico y autoconsumo colectivo). En todos los casos, la plataforma debe poder evolucionar en función de los desafíos relacionados a la energía y el conocimiento de la actividad humana es fundamental para evaluar, por un lado, la adecuación de la plataforma a las necesidades de los expertos en energía y por otro lado, para hacer evolucionar los modelos de la simulación.

\section{Dinamica y organización del programa de investigación tecnológico SMACH}

51 En este capítulo y, en un primer momento, proponemos explicitar la dinámica de emergencia del PIT SMACH. Para hacerlo, inspirándonos en la modelización del programa de investigación tecnológico genérica definida por Theureau (2009b, 2019), consideramos que la dinámica interna de la investigación SMACH es el resultado de la combinación de las siguientes tensiones :

- El impulso inicial corresponde a la definición de nuestro objeto de diseño. Desde el primer momento, abordamos la simulación como un problema de ayuda $\left[{ }^{10}\right]$ al usuario, hacer de manera que un experto pueda anticipar las transformaciones futuras vinculadas a la energía. No se trata entonces de utilizar un sistema multiagente para reemplazar al experto sino al contrario, se trata de utilizar las posibilidades de cálculo de la inteligencia artificial para ayudar al experto en energía en tomar sus decisiones de acción.

- Ese impulso inicial se apoya asimismo en nuestro posicionamiento teórico. Las hipótesis ontológicas y teóricas de la enacción y del curso de acción (dinámica de la actividad individual/colectiva, acoplamiento con el entorno, autonomía de los actores...) hacen que la actividad humana simulada deba ser realista.

- Este realismo in silico es considerado como posible a partir del acoplamiento estructural entre nuestras investigaciones sobre el conocimiento empírico de la actividad humana y las investigaciones en informática sobre los sistemas multiagentes. Este nudo central de la colaboración disciplinar es el que reúne luego todas las otras contribuciones.

- El compromiso en la investigación evoluciona de una etapa a otra del diseño, de un desafío al otro (factibilidad, desarrollo de la investigación, desarrollo del servicio) y enmarca la participación en las diferentes acciones de investigación.

- Las contribuciones disciplinares se estructuran alrededor de ese objetivo de realismo in silico y se apoyan en un observatorio de las situaciones reales de vida cotidiana y en situaciones realistas de experimentación.

- Las herramientas de la investigación (el taller) permiten producir resultados de investigación en términos de modelos sintéticos para el diseño y de metodologías de validación.

De este modo, la conjunción de esas diferentes tensiones es la que conduce la dinámica interna de la investigación tecnológica SMACH. La representamos más abajo (ver figura 16) retomando el formalismo en signo hexádico propuesto por Theureau (2009b, 2019): esta representación muestra la convergencia de las fuerzas para la producción de un 
resultado de investigación simbolizado aquí abajo (y en las demás figuras) por un punto naranja.

Figura 16: Dinámica interna de la investigación tecnológica SMACH

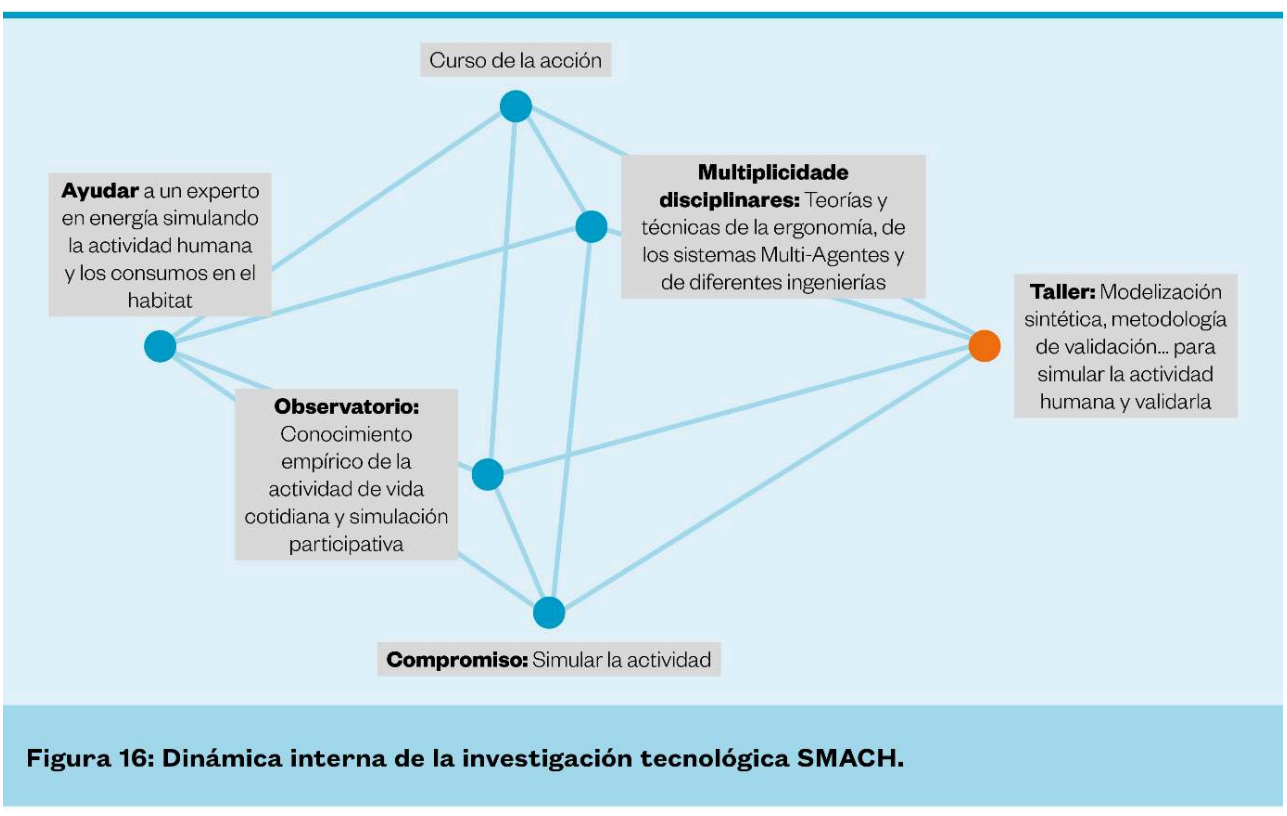

Figura 16: Dinámica interna de la investigación tecnológica SMACH

Es esta dinámica general la que determina el PIT SMACH y la que irá generando una organización en diferentes unidades significativas que estructuren la investigación tecnológica. Esas unidades son significativas de los diferentes niveles de acoplamiento estructural que existen entre la técnica y la descripción de la actividad humana. A continuación, especificamos esos niveles de organización de la investigación tecnológica SMACH.

El primer nivel de organización es el de los resultados significativos de la investigación tecnológica, representados en la figura 17 por un punto naranja. Dichos resultados, como vimos anteriormente, son el producto de la dinámica interna del PIT y corresponden principalmente en $\mathrm{SMACH}$ a resultados de modelización (el modelo sintético) o a resultados metodológicos (la simulación participativa). Corresponden a opciones de diseño tecnológico determinantes en el desarrollo de las preguntas de investigación. 
Figura 17: Los niveles de organización significativos de la investigación tecnológica SMACH

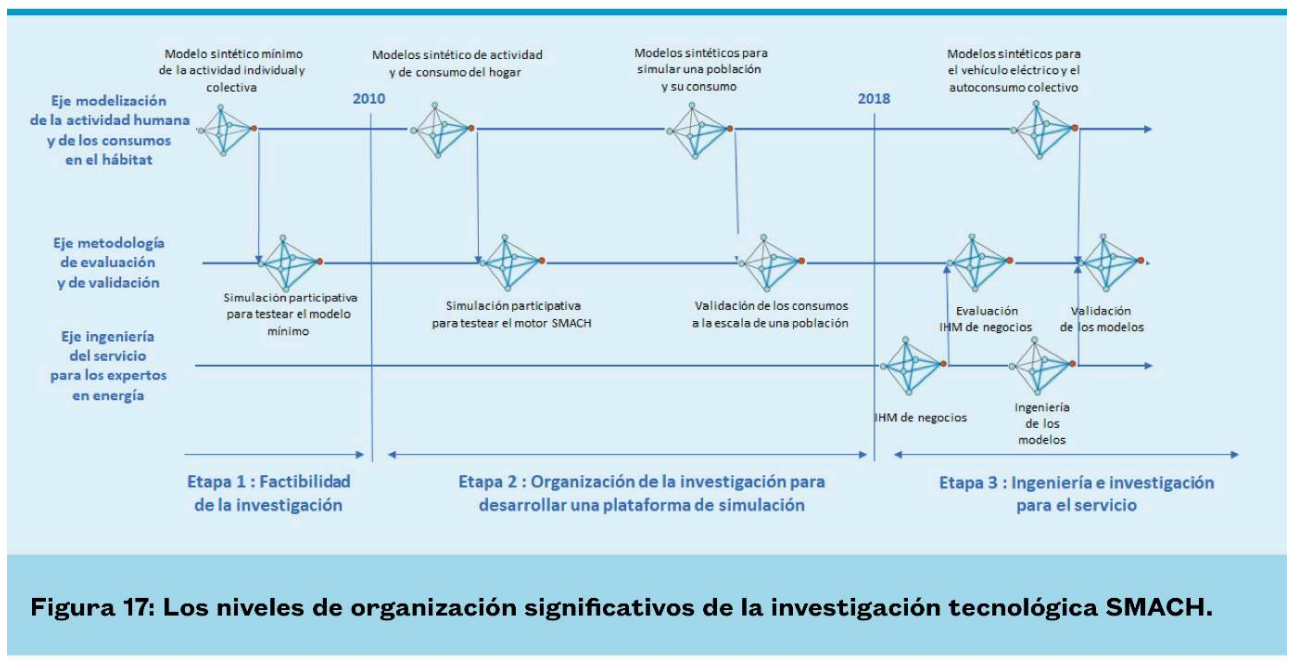

Figura 17: Los niveles de organización significativos de la investigación tecnológica SMACH

El segundo nivel de organización es el de las secuencias significativas de la investigación tecnológica. Este nivel de organización de la investigación da cuenta de la relación existente entre resultados significativos de modelización y resultados significativos metodológicos (las flechas en la figura 17). Hay aquí una fuerte dependencia entre esos dos tipos de resultados significativos. Por ejemplo, la secuencia "validez del modelo sintético limitado" corresponde a la articulación entre la simulación participativa y la modelización sintética limitada.

El último nivel de organización es el de la etapa de investigación tecnológica. Este nivel de organización corresponde al de los desafíos estratégicos del programa de investigación tecnológico. Por ejemplo, la etapa de factibilidad es el momento de verdad que debe hacer arrancar la investigación... o detenerla. Este nivel es esencial puesto que determina el tipo de compromiso en la investigación, y determina en parte, los niveles de organización encastrados que lo componen. Por ejemplo, el compromiso en la etapa 1 de factibilidad va a propiciar el diseño de un modelo sintético limitado y de un ensayo del método de simulación participativa. Se trata entonces de evaluar las posibilidades científicas y técnicas de este tipo de simulación. Como complemento, diremos que una etapa puede estar compuesta por una o varias secuencias significativas. Por ejemplo, la etapa de desarrollo de la plataforma está compuesta por dos secuencias significativas de investigación (secuencia "validez de la simulación de un hogar" y secuencia "validez de la simulación de una población").

Por ende, sobre la base de esos diferentes niveles de organización de la investigación, podemos precisar los criterios que definen el programa de investigación tecnológico SMACH:

- La relación orgánica con la actividad está presente en varios niveles del PIT SMACH. Cada resultado significativo de investigación (ya sea que se trate de las opciones de modelización o de las opciones metodológicas) se elabora en una relación estructural con el conocimiento empírico de la actividad humana. Además, las secuencias significativas de la investigación se construyen en torno a un bucle iterativo de modelización/validación que refuerza la relación orgánica con la actividad humana. 
- El poder heurístico del PIT SMACH es muy visible a nivel de las secuencias y de las etapas significativas de la investigación. La posibilidad de simular la actividad humana da lugar a la necesidad de simular a escala de un hogar, luego de una población y da también lugar a nuevas necesidades con la simulación del autoconsumo colectivo y el vehículo eléctrico. Esas posibilidades conducen finalmente a la necesidad de un servicio de generación de datos para los expertos en energía.

- El poder de crecimiento de la investigación se construye en la concatenación de los resultados significativos sobre los ejes modelización, metodología y servicio (ver figura 17) que abren cada vez nuevos interrogantes de investigación. Por ejemplo, la investigación para un modelo sintético evolucionó a lo largo del PIT pasando del modelo sintético limitado al modelo sintético avanzado, luego al modelo sintético a gran escala y después a los modelos para vehículo eléctrico y para el autoconsumo colectivo. Sucede lo mismo con el eje metodológico en el que la investigación estuvo referida a la definición de una instancia de test para validar, o bien la simulación de una actividad humana, o bien la simulación de los consumos de electricidad (simulación participativa, validación cuantitativa para el consumo de electricidad).

- Por último, tenemos el criterio de la eficacia técnico-organizacional cultural que ofrece criterios e índices de calidad tecnológica. Evaluamos la calidad a partir del eje metodológico en el que todos los modelos sintéticos pudieron ser evaluados en el marco de experimentaciones ergonómicas. También lo hacemos a partir del eje servicio en el que la calidad tecnológica se verifica en la evaluación de la ayuda aportada a los expertos en energía (utilizabilidad de la aplicación) así como en el servicio prestado (utilidad del servicio y de la aplicación) a esos expertos en respuesta a sus demandas de datos realistas de simulación.

58 En términos más generales, y como lo señalamos en las dinámicas de emergencia, el PIT $\mathrm{SMACH}$ es el fruto de una relación estructural con las distintas investigaciones empíricas y técnicas que lo nutrieron o que se convocaron (Durand, 2008; Theureau, 2009b). Por lo tanto, los modelos sintéticos se elaboran sobre la base de los conocimientos adquiridos en los distintos trabajos de investigación empírica acerca de la vida cotidiana en el hábitat (Guibourdenche, 2013; Guibourdenche et al., 2007; Haué, 2003). EL PIT SMACH también se nutrió de las investigaciones técnicas sobre la modelización y la simulación multiagente (Huraux et al., 2015; Plessis et al., 2014; Reynaud et al., 2017). El programa de investigación tecnológico SMACH dio también lugar a nuevos interrogantes para la investigación empírica y técnica. Es el caso, por ejemplo, del autoconsumo colectivo. Bonnardot (Bonnardot et al., 2021) se interroga sobre la anticipación de las experiencias colectivas futuras para compartir la energía producida localmente. Desde un punto de vista técnico, Albouys-Perrois (AlbouysPerrois et al., 2019) se interroga sobre la creación de mecanismos multiagentes para simular agrupamientos de autoconsumo colectivo. La dinámica interna del programa de investigación tecnológico SMACH, como todo PIT, es estructuralmente dependiente de su relación orgánica con investigaciones empíricas y técnicas.

Para concluir esta caracterización del programa de investigación tecnológico SMACH, abordaremos el tema de las colaboraciones de investigación e ingeniería. Cada etapa, cada secuencia, cada resultado significativo de la investigación tecnológica se construye alrededor de la definición de un objeto de investigación cuya unidad de diseño se apoya en las racionalidades limitadas de las diferentes disciplinas que lo nutre (Theureau, 2009a). Los modelos pivote de diseño (por ejemplo, el modelo sintético) y metodológico 
(por ejemplo, la simulación participativa) son los momentos privilegiados de esa construcción disciplinar. Más aún, en este aspecto, creemos que la investigación tecnológica remite a una transdiciplinariedad de diseño, es decir, al diseño de un objeto que transciende las diferencias disciplinares: ese objeto tiene una coherencia que no se encuentra en ninguna de las disciplinas tomadas por separado. El modelo sintético no hubiera existido sin los aportes de la ergonomía de la actividad y de la modelización multiagente. La simulación participativa no hubiera existido sin los aportes de la evaluación ergonómica, del diseño de interacción y de la metodología agente. La relación orgánica entre el conocimiento empírico de la actividad y el diseño técnico constituyen el cimiento de esa transdisciplinariedad de diseño. Esta transdiciplinariedad orientada a la actividad humana es constitutiva de nuestros objetos de investigación y lo es también de los conocimientos de ingeniería que implementamos.

\section{Conclusión}

El objetivo que nos propusimos al redactar este artículo fue el de describir un programa de investigación tecnológico específico apoyándonos en muchos años de trabajo en el marco de la investigación SMACH. Hemos insistido especialmente en la importancia de la relación orgánica con la actividad humana. Involucrarse en una investigación tecnológica significa entonces articular todos los momentos entre las dimensiones técnicas y el conocimiento empírico de la actividad humana. Articulación entre cada etapa de la investigación, entre cada secuencia y en el seno de cada resultado de la investigación, tomados de manera independiente uno del otro. Es ese movimiento, construido en una relación orgánica con diferentes programas de investigación científica (empírica y técnica), el que permite responder a las necesidades de simulación cada vez más grandes. Y es también ese movimiento, sin lugar a dudas, el que sostiene la calidad tecnológica de la situación concebida/diseñada.

61 Para terminar, y retomando las palabras de Pinsky (Pinsky, 1990a), con este texto, aspiramos a situarnos en una reflexión sobre el diseño, a hacer una pequeña contribución a una teoría de la práctica. Describimos aquí el PIT específico "simular la actividad humana in silico", basándonos en la investigación SMACH. Un trabajo futuro sería comparar esta investigación con otras que integren la actividad humana a la simulación a fin de precisar las reglas de ese tipo de programa de investigación tecnológico específica. Por supuesto, al comparar diferentes PIT específicos, contribuimos también a una reflexión más general sobre el diseño, y en especial, sobre el programa de investigación tecnológico genérica del curso de acción.

\section{BIBLIOGRAFÍA}

Albouys-Perrois, J., Sabouret, N., Haradji, Y., Schumann, M., \& Inard, C. (2019). A Co Simulation Of Photovoltaic Power Generation And Human Activity For Smart Building Energy Management And 
Energy Sharing. Proceeding of 16th IBPSA International Conference \& Exhibition Building Simulation 2019. Florence, Italie.

Barcellini, F., Van Belleghem, L., \& Daniellou, F. (2013). Les projets de conception comme opportunité de développement des activités. In P. Falzon (Ed.), Ergonomie constructive (pp. 191206). Paris: Presses Universitaires de France.

Béguin, P., \& Weill-Fassina, A. (1997). La Simulation en ergonomie : connaitre, agir et interagir. Toulouse: Octarès.

Bonnardot, Z., Vial, S., Salembier, P., Prieur, E., \& Haradji, Y. (2021). Créer des interactions humaines réalistes par le design : le cas de l'autoconsommation collective pour la transition énergétique (Texte accepté). Actes Du 55ème Congrès de La SELF, Paris, 11-13 Janvier 2021.

Delenne, B. (2018). Eléments de validation des courbes de charge simulées par SMACH. In Document interne EDF. Mémo E74/18/010/A.

Dugdale, J., Pavard, B., \& Soubie, J.-L. (2000). A Pragmatic Development of a Computer Simulation of an Emergency Call Centre. In G. Dieng, R. Giboin, A., Karsenty, L., \& De Michelis (Eds.), Proceedings of the 4th International Conference on the Design of Cooperative Systems (pp. 241-256). Amsterdam: IOS Press.

Durand, M. (2008). Un programme de recherche technologique en formation des adultes. Éducation et Didactique, 2(3), 97-121. https://doi.org/10.4000/educationdidactique.373

Ferber, J. (1995). Les Systèmes multi-agents : vers une intelligence collective. InterEditions.

Guibourdenche, J. (2013). Préoccupations et agencements dans les contextes d'activité domestique : Contribution à la conception de situations informatiques diffuses, appropriables et énergétiquement efficaces. Thèse de doctorat, Institut de psychologie Lyon2.

Guibourdenche, J., Salembier, P., Poizat, G., Haradji, Y., \& Galbat, M. (2007). A Contextual Approach to Home Energy Management Systems Automation in Daily Practices. Proceedings of the European Conference on Cognitive Ergonomics 2015. https://doi.org/10.1145/2788412.2788436

Guyot, P. (2006). Simulations multi-agents participatives. Faire interagir agents et humains pour explorer, modéliser et reproduire les comportements collectifs. Thèse de doctorat, Université Paris 6.

Haradji, Y., \& Faveaux, L. (2006). Évolution de notre pratique de conception (1985-2005). Activites, 03(1). https://doi.org/10.4000/activites.1852

Haradji, Y., Guibourdenche, J., Reynaud, Q., Poizat, G., Sabouret, N., Sempé, F., Huraux, \& Galbat, M. (2018). De la modélisation de l'activité humaine à la modélisation pour la simulation sociale : entre réalisme et fécondité technologique. Activites, 15(1). https://doi.org/10.4000/activites.3106

Haradji, Y., Poizat, G., \& Sempé, F. (2012). Human Activity and Social Simulation. Advances in Applied Human Modeling and Simulation. CRC Press.

Haué, J.-B. (2003). Conception d'interfaces grand public en termes de situations d'utilisation : le cas du Multi-Accès. Thèse de doctorat, Université de Technologie de Compiègne.

Huraux, T., Sabouret, N., \& Haradji, Y. (2015). Study of Human Activity Related to Residential Energy Consumption Using Multi-level Simulations. Proceedings of the International Conference on Agents and Artificial Intelligence, 133-140. https://doi.org/10.5220/0005197401330140

Jeffroy, F., \& Lambert, I. (1992). An ergonomics framework for user activity centred software design. Human Factors in Information Technology, 9, 43-92. https://doi.org/10.1016/

B978-0-444-89301-7.50009-5 
Kashif, A., Ploix, S., Dugdale, J., \& Le, X. H. B. (2013). Simulating the dynamics of occupant behaviour for power management in residential buildings. Energy and Buildings. https://doi.org/ 10.1016/j.enbuild.2012.09.042

Koyré, A. (1971). Études d'histoire de la pensée philosophique. Gallimard.

Le Page, C. (2017). Simulation multi-agent interactive: engager des populations locales dans la modélisation des socio-écosystèmes pour stimuler l'apprentissage social. Université Pierre et Marie Curie, Paris.

Lefèvre, A. (2016). Simulation sociale et simulacre structural. Variations, (19), 1-23. https:// doi.org/10.4000/variations.720

Norman, D. A., \& Draper, S. W. (1986). User Centered System Design. New Perspectives on HumanComputer Interaction. CRC Press.

Pavard, B. (2002). Complexity paradigm as a framework for the study of cooperative systems. Revue d'intelligence Artificielle, 16(4-5), 419-442. https://doi.org/10.3166/ria.16.419-442

Pinsky, L. (1990a). Définir l'ergonomie comme une technologie. Communication Au XXVIe Congrès de La Société d'Ergonomie de Langue Française, Montréal., pp. 1-10.

Pinsky, L. (1990b). User activity centered design. In Elsevier (Ed.), Work with display units (L. Berling (pp. 119-150). North-Holland.

Pinsky, L., \& Theureau, J. (1984). Paradoxe de l'ergonomie de conception et logiciel informatique. Revue Des Conditions de Travail, 9, 1-15.

Pinsky, L., \& Theureau, J. (1987). L'étude du cours d'action. Collection d'Ergonomie et de Neurophysiologie du Travail CNAM N ${ }^{\circ} 88$. Paris.

Plessis, G., Amouroux, É., \& Haradji, Y. (2014). Coupling occupant behaviour with a building energy model-A FMI application. Proceedings of the 10th International ModelicaConference, 321-326. https://doi.org/10.3384/ECP14096321

Poizat, G., Durand, M., \& Theureau, J. (2016). The challenges of activity analysis for training objectives. Le Travail Humain, 79(3), 233. https://doi.org/10.3917/th.793.0233

Reynaud, Q., Haradji, Y., Sempé, F., \& Sabouret, N. (2017). Using Time Use Surveys in Multi Agent based Simulations of Human Activity. Proceedings of the 9th International Conference on Agents and Artificial Intelligence, 67-77. https://doi.org/10.5220/0006189100670077

Theureau, J. (2006). Le cours d'action : méthode développée. Toulouse: Octarès.

Theureau, J. (2009a). Comment l'interdisciplinarité peut-elle être un rassemblement fécond des ignorants? In La mise à l'épreuve. Le transfert des connaissances scientifiques en questions (QUAE, (pp. 121-139). Versailles.

Theureau, J. (2009b). Le cours d'action : méthode réfléchie. Toulouse: Octarès.

Theureau, J. (2011). Appropriations 1, 2, 3. Appropriation, Incorporation, In-culturation. Retrieved from http://www.coursdaction.fr/02-Communications/2011-JT-C136.pdf

Theureau, J. (2019). Cerisy : La construction de programmes de recherche technologique en relation organique avec des recherches en sciences humaines \& sociales. Cerisy.

Van Belleghem, L. (2018). La simulation de l'activité en conception ergonomique : acquis et perspectives. Activites, 15(1), 0-22. https://doi.org/10.4000/activites.3129

Varela, F. J. (1989). Autonomie et connaissance : essai sur le vivant. Éditions du Seuil. 
Vinck, D., \& Laureillard, P. (1995). Coordination par les objets dans les processus de conception. Journées CSI - "Représenter, Coordonner, Attribuer," 13. Retrieved from https://hal.archivesouvertes.fr/hal-00134434

Winograd, T., \& Flores, F. (1986). Understanding computers and cognition : a new foundation for design. Norwood: Ablex.

\section{NOTAS}

1. El significado del acrónimo SMACH ha evolucionado. En sus comienzos significaba Simulación Multiagente de los Comportamientos Humanos. En la actualidad, significa Simulación Multiagente de la Actividad Humana y de los Consumos en el Hábitat.

2. IHM significa aquí Interacción Humano-Máquina y se centra en la ayuda que esa IHM aporta en su interacción con la actividad del usuario.

3. El diagnóstico/pronóstico del ergónomo se basa sobre los criterios de ayuda (volumen de trabajo, facilidad de la utilización, utilidad, etc.) y sobre la descripción que hace de la actividad humana.

4. Utilizaremos la mayoría de las veces la abreviatura PIT para hablar del programa de investigación tecnológico del "curso de acción" (en francés: PRT Programme de Recherche Technologique). Los términos programa de investigación tecnológico específica, PIT SMACH, investigación tecnológica SMACH, serán utilizados en este texto como términos equivalentes.

5. El diseño del modelo sintético y su relación con el análisis empírico de la actividad de vida cotidiana están presentados en Haradji et al. (2018)

6. Un agente tendrá la posibilidad de realizar una acción en función de períodos preferenciales (por ejemplo, desear dormir por la noche), de preferencias sobre las acciones (preferir leer más que hacer las tareas del hogar), de reacciones al entorno (tener calor o frío), de coordinaciones con otros agentes (salir para hacer un paseo), etc. El conjunto de esas limitaciones está calculado para un período de un minuto y determina el peso respectivo (valor numérico) de cada acción para cada agente: una acción puede entonces ser activada, continuada, interrumpida...

7. Las palabras en cursiva corresponden a palabras de la figura 9.

8. «Une Bretagne d'avance» - Programa que se realiza a nivel de la región de Bretaña en Francia.

9. Los trabajos presentados en este capítulo están en curso y no pueden ser desarrollados con el mismo nivel de precisión con el que fueron presentados en los capítulos anteriores.

10. Las palabras en cursiva corresponden a los componentes del signo hexádico presentados en la figura 15

\section{RESÚMENES}

SMACH (Simulación Multiagente de la Actividad Humana y los Consumos en el Hábitat) es una plataforma de simulación que se inserta en un marco de investigación y desarrollo para la EDF (Électricité de France - Empresa nacional de electricidad de Francia). Tiene por objeto utilizar la simulación para anticipar y reducir el consumo de energía en diferentes mallas de análisis (hábitat, barrio, ciudad, país). Con este texto, nos propusimos describir qué es un programa de investigación tecnológico curso de acción, basándonos para ello en muchos años de trabajo de 
investigación SMACH. Insistimos principalmente en la importancia de la relación orgánica entre técnica y actividad humana y mostramos la evolución de esa relación orgánica a lo largo de las diferentes etapas que caracterizan a este programa de investigación tecnológico. Este artículo puede ser considerado como una base para generalizar y precisar los criterios efectivos de validación de un programa de investigación tecnológico y como una contribución a una reflexión sobre el diseño en ergonomía.

SMACH (Simulação Multiagente da Atividade Humana e do Consumo na Habitação) é uma plataforma de simulação que faz parte da investigação e desenvolvimento da EDF. Tem como objetivo utilizar a simulação para a antecipação e redução do consumo de energia em diferentes locais de análise (habitação, distrito, cidade, país). o nosso objetivo, com este texto, é caracterizar o que é um programa de pesquisa tecnológica repleto de ação, com base em vários anos de pesquisa SMACH. Destacamos principalmente a importância da relação orgânica entre a técnica e a atividade humana e mostramos a evolução desta relação orgânica através das diferentes etapas que caracterizam este programa de investigação tecnológica. Este artigo pode ser visto como uma base para generalizar e especificar os critérios efetivos de validação de um programa de pesquisa tecnológica, como uma contribuição para uma reflexão sobre design em ergonomia.

SMACH (Simulation Multi-agent de l'Activité humaine et des Consommations dans l'Habitat) est une plateforme de simulation qui s'inscrit pour EDF dans un cadre de recherche et développement. Elle vise à utiliser la simulation pour l'anticipation et la réduction des consommations d'énergie à différentes mailles d'analyse (habitat, quartier, ville, pays). Notre objectif, avec ce texte, est de caractériser ce qu'est un programme de recherche technologique cours d'action en nous appuyant pour cela sur plusieurs années de travaux de recherche SMACH. Nous insistons principalement sur l'importance de la relation organique entre technique et activité humaine et nous montrons l'évolution de cette relation organique au fur et à mesure des différentes étapes qui caractérisent ce programme de recherche technologique. Cet article peut être vu comme une base pour généraliser et préciser les critères effectifs de validation d'un programme de recherche technologique, comme une contribution à une réflexion sur la conception en ergonomie.

SMACH (Multi-agent Simulation of Human Activity and Household Consumption) is a simulation platform within EDF's R\&D framework. It uses simulation to anticipate and reduce energy consumption at different places of analysis (home, district, town, country). With this paper we aim to explain what the course of action technological research program involves, and to this end we refer to several years of SMACH research. We essentially focus on the importance of the organic relationship between technology and human activity, and we show how this organic relationship evolves over the different stages that this technological research program involves. This paper may be viewed as a basis for generalizing and clarifying the criteria required to validate such a program and as a contribution towards reflection on design in ergonomics. 
ÍNDICE

Palabras claves: programa de investigación tecnológico, modelo sintético, diseño, simulación participativa, curso de acción, análisis de la actividad, sistema multiagente

Keywords: technological research program, synthetic model, design, participatory simulation, course of action, activity analysis, multi-agent system

Palavras-chave: programa de pesquisa tecnológica, modelo sintético, design, simulação participativa, curso de ação, análise da atividade, sistema multiagente

Mots-clés: programme de recherche technologique, modèle synthétique, conception, simulation participative, cours d'action, analyse de l'activité, système multi-agent

\section{AUTOR}

\section{YVON HARADJI}

EDF Lab Paris Saclay. 7 boulevard Gaspard Monge. 91120 Palaiseau. France yvon.haradji@gmail.com 